\title{
Hydrodynamic Modeling and Performance Analysis of Bio-inspired Swimming
}

\author{
Mehdi Ghommem ${ }^{1 *}$, George Bourantas², Adam Wittek², Karol Miller², and Muhammad R. Hajj ${ }^{3}$ \\ ${ }^{I}$ Department of Mechanical Engineering, American University of Sharjah, Sharjah, United Arab Emirates \\ ${ }^{2}$ Intelligent Systems for Medicine Laboratory, The University of Western Australia, 35 Stirling Highway, Perth, WA 6009, \\ Australia \\ ${ }^{3}$ Department of Civil, Environmental, and Ocean Engineering, Stevens Institute of Technology, New Jersey, United States \\ of America
}

\begin{abstract}
We simulate thrust generation, lateral forces, and vorticity patterns in the wake of a swimming deformable fish-like body having different fin configurations. The simulation code is validated by comparing hydrodynamic loads and velocity profiles with published experimental and numerical results. The hydrodynamic performance of undulatory body is based on evaluation of generated loads versus required kinematics to achieve thrust-based swimming. The results show significant dependence of the thrust generation and vorticity pattern of the wake on the Strouhal number. Analysis of lateral oscillations of the tail reveals the existence of an optimal lateral oscillation amplitude that produces positive thrust. Simulations with a flapping caudal fin show significant improvement in thrust generation and transition from drag-based to thrust-based swimming at lower Strouhal numbers. Furthermore, controlling the flapping frequency of the caudal fin yields further enhancement in the hydrodynamic performance. This enhancement is associated with the transition from the aligned arrangement of alternating vortices to a deflected wake producing a momentum surfeit in the near wake region. Simulations with rigid pectoral fins on the generation of hydrodynamic loads show that a reduction in thrust production. This degradation in the propulsive performance is associated with the flow separation on the fish body.
\end{abstract}

Keywords: fish-like swimming, hydrodynamic performance, wake structure, undulatory motion, flapping caudal fin; pectoral fin.

\section{Introduction}

Sophisticated autonomous underwater vehicles (AUVs) can substitute human divers and conventional submersible systems deploying rotary propellers in performing meticulous tasks in marine environments. These tasks include monitoring, inspection of underwater structures (e.g., risers and foundations of offshore oil platforms and wind turbines), rescue and search operations after natural catastrophes, surveillance of aquatic animals in aquacultures, and tracking of small and hidden objects in confined aquatic spaces. To successfully accomplish these missions, the design of these vehicles needs to satisfy specific performance requirements such as navigation autonomy including target recognition, obstacle avoidance, detection capabilities and awareness of the environment, agile maneuverability, long endurance, low acoustic noise or silent swimming, and a good stability. As such, researchers and engineers have been inspired by

* Corresponding author: mghommem@aus.edu 
swimming species, especially their body morphology, fin shape, and kinematics, to develop an understanding of the associated locomotion mechanics and come up with different functional designs [19]. Fish rely mainly on two locomotion modes in swimming, namely, body and/or caudal fin (BCF) locomotion, and median and/or paired fin (MPF) locomotion [10]. The BCF swimmers generate thrust using lateral movements (bending) of their body and caudal fin resulting in undulatory/wavy or oscillatory motion. The MPF aquatic animals swim by controlling the movement and rotation of their median and pectoral fins and has been observed to be suitable for swimming at slow speeds, enabling superior maneuverability and reaching higher propulsive efficiency. On the other hand, the BCF locomotion mode has the capability to generate greater thrust and achieve higher speeds and accelerations. The latter locomotion mode is used by about $85 \%$ of the fish species [9]. Any of these modes or a combination of the two can be replicated to develop robotic systems that could outperform live fish and used in various engineering applications [1-11].

Inspired by fish swimming and exploiting recent advances in bio-robotics and mechatronics research, several research groups have successfully built and tested the performance of fish-like robots [1-7]. The development of these robots involve the study of several aspects including locomotion pattern, kinematics, hydrodynamics, motion control, body morphology and material. Various bio-inspired robots have been reported in the literature $[1-8,11]$. These robots differ in terms of the propulsion mechanisms, which include a single joint with flapping fin or tail, multi-joint using a serially linked motor, or a soft flexible body made of smart material, the locomotion mode BCF or MPF, the buoyancy control system, the size, the autonomy level, and the swimming capabilities.

The aforementioned performance requirements of the robotic fish can be assessed at the earliest stages of design through the use of mathematical models and computational tools. Advances in computer hardware and software have enabled reduction in the computational burden associated with the numerical integration of highly nonlinear partial differential equations governing the coupled fluid-structure interaction associated with the operation of the robot. Several research studies have been recently devoted to model and simulate fish-like swimming using unsteady Navier-Stokes equations with the goal of investigating the hydrodynamic performance of fish undergoing undulatory motion [12-19]. Bergmann and Iollo [12] used a two-dimensional laminar flow solver to examine fluid dynamics aspects of different BCF swimming modes. Each mode was represented by specific body shape and periodic deformation law. They also analyzed the power requirements of the fish to perform abrupt turns and maneuvers. They demonstrated the exploitation by the fish of the surrounding previously shed vortices to reach energy savings for the locomotion. Li et al. [13] conducted numerical simulations to study the hydrodynamics of a flexible tunalike fish with an undulating body and oscillating caudal fin. They developed a fully coupled computational model accounting for the fish-fluid interactions and analyzed different dynamic features of the selfpropelled swimming of the fish such as the generated hydrodynamic forces, the swimming speed, and wake vortex structure under varying kinematics. The swimming performance in terms of power requirements and swimming speed was observed to improve when increasing the oscillating amplitude of the caudal fin. This improvement is associated with a tilting effect of the tail making the generated pressure difference more aligned along the thrust direction. Khalid et al. [14] used the immersed boundary method to simulate flow past a single and two fish with undulating body and placed behind each other at varying distances. Swimming in proximity of other fish is found to be more beneficial for the upstream fish (leading) due to the wake vortices splitting caused by the rear fish (follower). On the other hand, the energetic gain of the rear fish from the hydrodynamic interactions is observed to be significantly dependent on the Strouhal number. In a recent paper, Khalid et al. [16] studied the nonlinear hydrodynamic interactions of two fish in group arrangements. They computed the Fourier spectra of the hydrodynamic forces and observed the presence of higher-order harmonics indicating a nonlinear coupling between the undulating frequencies of the wavy motions of the fish. Xia et al. [18] developed a three-dimensional flow solver to study the effect of the head swing motion on the fishlike robot swimming performance. They found that superior 
hydrodynamic performance can be achieved when selecting properly the leading and trailing edges of the head as indicated by the induced flow structures.

Computational power and time required to perform three-dimensional hydrodynamic simulations to discern specific interrelated effects of causal mechanisms associated with fish-like swimming hinder the capability to perform parametric and sensitivity analysis. In contrast, two-dimensional hydrodynamic simulations could provide useful insights of underlying phenomena of fish-like swimming that may be directly applicable to real-life three-dimensional problem as demonstrated in several recent research studies [14, 16, 19-22]. Zhao and Dou [19] conducted a numerical simulation of the impact of the structural connection between a fish-like body and caudal fin on the swimming performance of a single fish. Their results showed that a disconnected structural configuration (i.e., detaching the caudal fin from the main body) leads to pronounced improvement in thrust generation and increases the propulsive efficiency in comparison with a connected structural configuration. They analyzed the flow mechanisms responsible for the observed enhancement in the undulatory propulsion. Gao and Triantafyllou [20] simulated a tandem arrangement of two self-propelled flexible fish bodies undergoing wavy motion and equipped with a rigid caudal fin undergoing prescribed heave and pitch motions. They considered a gap distance of $5 \%$ of the body length separating the main body from the caudal fin and implemented PID controllers to enforce self-propulsion and maintain straight-line swimming mode. They demonstrated the importance of caudal fin control to lower significantly the energy required to secure self-propulsion by reducing the interference with vortices generated by the upstream fish and enhancing the thrust production. Independent pitch control of the caudal fin, based on applying vorticity control mechanisms, led to more than $50 \%$ reduction in the energy required to achieve self-propulsion.

In this paper, we conduct numerical simulations to investigate the locomotive/propulsive roles of tail undulation and caudal fin flapping in the swimming performance and hydrodynamic loads generation of fish. We identify the key kinematics parameters for design enhancement of bio-inspired underwater vehicles in terms of thrust production. The objective of the present study is to extend the research on numerical simulations of bio-inspired swimming and contribute to the development and validation of a computational model of fluid flow around a deforming fish-like body by combining the newly developed meshless [23] and immersed boundary methods (at the Intelligent Systems for Medicine Laboratory of the University of Western Australia) and the numerical investigation of the impact of fish kinematics and morphology, namely undulatory body with and without caudal and pectoral fins, on the propulsive performance dictated by thrust force generation. The remainder of the paper is organized as follows. In Section 2, we present the numerical methodology and the benchmark cases used for validation. Section 3 is concerned with the study of hydrodynamic performance of fish-like swimming. In Section 4, we summarize the main findings of the numerical investigation and conclude with some remarks and recommendations for the design of robotic fish.

\section{Numerical Methodology and Code Verification}

One particular aspect of this effort is the presented numerical approach, which demonstrates the capability of combining explicit dynamics meshless point collocation using Discretization Corrected Particle Strength Exchange [23] with immersed boundary method to solve the vorticity stream-function formulation of the incompressible Navier-Stokes equations and compute the fluid forces around moving/deforming bodies. 


\subsection{Governing equations and numerical scheme}

To solve the governing equations of incompressible fluid flow around a moving/deforming body, we consider a rectangular computational Eulerian domain $\Omega$, which involves an immersed body described in the form of a closed curve $\partial \Omega^{L}$, as shown in Fig. 1. For 2D cases, using the stream function-vorticity equation presented in Eq. (1) and (2) to simulate the incompressible flow is preferable since the continuity equation or conservation of mass is inherently satisfied. The governing flow equations are solved on the Eulerian grid, following the method described by Bourantas et. al. [23], and the flow field is adjusted so that the prescribed boundary conditions for the velocity on the immersed body (Lagrangian nodes) are satisfied.

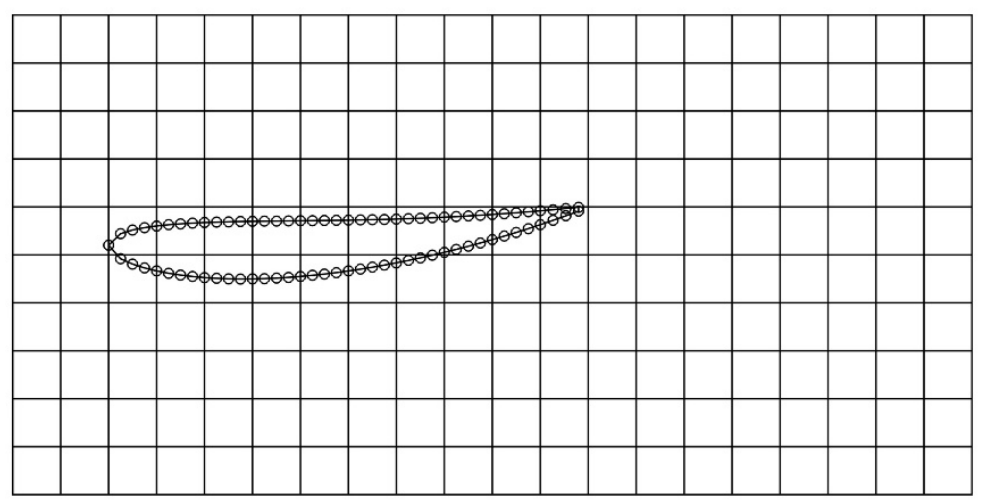

Fig. 1. Schematic of the Cartesian grid (Eulerian) and the immersed body (circular dots).

The immersed boundary is accounted for by including an additional term in the flow equations. The additional term can be considered as a vorticity correction, and is introduced in the stream function equation given by Eq. (2). Therefore, the governing equations describing the fluid motion with the use of immersed boundary (IB) method are written in their stream function-vorticity formulation as

$$
\begin{aligned}
& \rho\left(\frac{\partial \omega}{\partial t}+\frac{\partial \psi}{\partial y} \frac{\partial \omega}{\partial x}-\frac{\partial \psi}{\partial x} \frac{\partial \omega}{\partial y}\right)=\mu \nabla^{2} \omega \\
& \nabla^{2} \psi=-\omega+\delta \omega^{*}
\end{aligned}
$$

subject to the following boundary and initial conditions $(t=0)$

$$
\begin{aligned}
& \boldsymbol{u}(\boldsymbol{X}(s))=\boldsymbol{U}_{L} \text { on } \partial \Omega^{L} \\
& \boldsymbol{u}=\boldsymbol{u}_{0} \\
& \boldsymbol{\omega}=\boldsymbol{\omega}_{\mathbf{0}}=\boldsymbol{\nabla} \times \boldsymbol{u}_{\mathbf{0}}
\end{aligned}
$$

with $\boldsymbol{u}=\left(u_{x}, u_{y}\right), \psi$ and $\omega$ being the velocity vector, stream function, and vorticity, respectively, while $\rho$ and $\mu$ denote the fluid density and viscosity, respectively. The additional term $\delta \omega^{*}$ in Eq. (2) represents the vorticity source, while $\boldsymbol{x}=(x, y)$ and $\boldsymbol{X}(s)$ (the immersed object can be viewed as a parametric curve $\boldsymbol{X}(s)$, where $s$ is the parameter) stand for the Eulerian and Lagrangian coordinates, respectively. The velocity at the Lagrangian points can be interpolated from the velocity at the fluid (Eulerian mesh) points as

$u(X(s, t))=\int u(x, t) \delta(x-X(s, t)) d x$

where $\delta(\boldsymbol{x}-\boldsymbol{X}(s, t))$ is the Dirac delta function representing the interaction between the fluid and the immersed boundary. 


\subsection{Boundary Condition Enforced Immersed Boundary (BCE-IB) method}

We numerically solve the strong form of the governing equations using the Euler explicit time integration scheme for the transient terms, and a collocation method to compute spatial derivatives. The Euler scheme is easy to apply, but results in small time steps, especially for high Reynolds numbers. Following Bourantas et al. [23,24], we apply the explicit Euler scheme to discretize the time derivative in Eq. (1). We compute the predicted vorticity $\widetilde{\omega}$, using the values for the stream function $\psi^{n}$ and vorticity $\omega^{n}$ at the previous time step (we use the notation $\omega^{n+1}=\omega\left(t^{n+1}\right)$ and $\psi^{n+1}=\psi\left(t^{n+1}\right)$, where $t^{n+1}=t^{n}+\delta t$, through the equation

$$
\rho\left(\frac{\widetilde{\omega}-\omega^{n}}{\Delta t}\right)=-\rho\left(\frac{\partial \psi^{n}}{\partial y} \frac{\partial \omega^{n}}{\partial x}+\frac{\partial \psi^{n}}{\partial x} \frac{\partial \omega^{n}}{\partial y}\right)+\mu \nabla^{2} \omega^{n}
$$

We compute the predicted stream function $\tilde{\psi}$ field value by solving the Poisson equation

$$
\nabla^{2} \tilde{\psi}=-\widetilde{\omega}
$$

The spatial derivatives in Eqs. (7) and (8) are computed using the Discretization Corrected Particle Strength Exchange (DC PSE) interpolation scheme [25-27] - one of the most accurate and efficient point collocation methods to compute spatial derivatives. The predicted velocity $\tilde{\boldsymbol{u}}=(\tilde{u}, \tilde{v})$ field value is calculated as

$$
\begin{gathered}
\tilde{u}=\frac{\partial \tilde{\psi}}{\partial y} \\
\tilde{v}=-\frac{\partial \tilde{\psi}}{\partial x}
\end{gathered}
$$

We correct the velocity field, such that the prescribed velocity boundary conditions on the immersed boundary are satisfied. The corrected/updated velocity field $\boldsymbol{u}^{n+1}$ is written as

$$
\boldsymbol{u}^{n+1}=\widetilde{\boldsymbol{u}}+\delta \boldsymbol{u}^{*}
$$

with $\delta \boldsymbol{u}^{*}=\left(\delta u^{*}, \delta v^{*}\right)$ being the velocity correction. Accordingly, the corrected/updated vorticity value $\omega^{(n+1)}$ is written as

$$
\omega^{n+1}=\widetilde{\omega}+\delta \omega^{*}
$$

with $\delta \omega^{*}$ being the vorticity correction. The updated vorticity field $\omega^{n+1}$ is computed as

$$
\widetilde{\omega}+\delta \omega^{*}=\frac{\partial u^{n+1}}{\partial y}-\frac{\partial v^{n+1}}{\partial x}
$$

and the vorticity correction $\delta \omega^{*}$ is written as

$\delta \omega^{*}=\boldsymbol{\nabla} \times \delta \boldsymbol{u}^{*}=\frac{\partial(\delta u)}{\partial y}-\frac{\partial(\delta v)}{\partial x}$

Once the velocity correction is calculated, the updated velocity $\boldsymbol{u}^{n+1}$ and vorticity $\omega^{n+1}$ field values are calculated using Eqs. (11) and (12), respectively. 
The velocity correction results in the corrected velocity $\boldsymbol{u}^{n+1}$ equal to the prescribed velocity $\boldsymbol{U}_{B C}$ on the Lagrangian points on the boundary. When no-slip boundary conditions are prescribed the velocity $\boldsymbol{U}_{B C}$ is set equal to zero. Therefore, the velocity correction $\delta \boldsymbol{u}^{*}$ interpolated at the Lagrangian points $(\boldsymbol{u}(\boldsymbol{X}(s), t))$, accounts for the prescribed boundary velocity $\boldsymbol{U}_{B C}$ on the immersed boundary, and it is distributed from the virtual boundary flux $\delta \boldsymbol{U}$ through the delta function interpolation

$$
\delta \boldsymbol{u}^{*}(\boldsymbol{x}, t)=\int \delta \boldsymbol{U}(\boldsymbol{X}(s), t) \delta(\boldsymbol{x}-\boldsymbol{X}(s, t)) d s
$$

The immersed boundary is represented by a set of points $\boldsymbol{X}^{j} \quad(j=1,2, \ldots, M)$ (Lagrangian points), and the fluid by a set of fixed uniform Cartesian grid $\boldsymbol{x}_{i}(i=1,2, \ldots, N)$, with mesh spacing $h_{x}=h_{y}=h$. Furthermore, the Dirac function $\delta(\boldsymbol{x}-\boldsymbol{X}(s, t))$ is approximated by a continuous kernel distribution $D\left(\boldsymbol{x}_{i}-\boldsymbol{X}^{j}\right)$ given as

$$
D_{i j}=D\left(\boldsymbol{x}_{i}-\boldsymbol{X}^{j}\right)=\left(\frac{1}{h} \delta\left(\frac{x_{i}-X^{j}}{h}\right)\right)\left(\frac{1}{h} \delta\left(\frac{y_{i}-Y^{j}}{h}\right)\right)
$$

with the kernel $\delta(r)$ proposed by Lai and Peskin [25]

$$
\delta(r)=\left\{\begin{array}{lr}
\frac{1}{8}\left(3-2|r|+\sqrt{1+4|r|-4 r^{2}}\right) & |r| \leq 1 \\
\frac{1}{8}\left(5-2|r|-\sqrt{-7+12|r|-4 r^{2}}\right) & 1<|r| \leq 2 \\
0 & |r|>2
\end{array}\right.
$$

Eq. (15) is written in a discrete form as:

$$
\delta \boldsymbol{u}^{*}\left(\boldsymbol{x}_{i}, t\right)=\sum_{j} \delta \boldsymbol{U}\left(\boldsymbol{X}^{j}, t\right) D\left(\boldsymbol{x}_{i}-\boldsymbol{X}^{j}\right) \Delta s_{j}
$$

with $\delta \boldsymbol{u}^{*}\left(\boldsymbol{x}_{i}, t\right)$ being the velocity correction at the $i^{\text {th }}$ Eulerian point, and $\Delta s_{j}$ the $j^{\text {th }}$ boundary segment length. The unknowns in Eq. (18) are the fictitious boundary fluxes $\delta \boldsymbol{U}\left(\boldsymbol{X}^{j}, t\right)$. To satisfy the prescribed boundary condition, the velocity $\boldsymbol{U}\left(\boldsymbol{X}^{j}, t\right)$ at the Lagrangian points, projected from the corrected fluid velocity field $\boldsymbol{u}^{(n+1)}$ through the smooth delta function $D_{i j}$, must be equal to the prescribed boundary velocity $\boldsymbol{U}_{B C}\left(\boldsymbol{X}^{j}, t\right)$; that is,

$$
\boldsymbol{U}\left(\boldsymbol{X}^{j}, t\right)=\sum_{i} \boldsymbol{u}^{(n+1)}\left(\boldsymbol{x}_{i}, t\right) D\left(\boldsymbol{x}_{i}-\boldsymbol{X}^{j}\right) h^{2}
$$

In Eq. (19), $\boldsymbol{u}^{n+1}\left(\boldsymbol{x}_{i}, t\right)$ is the corrected velocity at the $i^{t h}$ Eulerian point, expressed as the sum of the tentative velocity $\widetilde{\boldsymbol{u}}$ and velocity correction $\delta \boldsymbol{u}^{*}$, as shown in Eq. (11). Substituting Equations. (11) and (18) into Equation (19) we get 


$$
\boldsymbol{U}\left(\boldsymbol{X}^{j}, t\right)=\sum_{i} \widetilde{\boldsymbol{u}} D\left(\boldsymbol{x}_{i}-\boldsymbol{X}^{j}\right) h^{2}+\sum_{i} \sum_{k} \delta \boldsymbol{U}\left(\boldsymbol{X}^{j}, t\right) D\left(\boldsymbol{x}_{i}-\boldsymbol{X}^{k}\right) \Delta s_{k} D\left(\boldsymbol{x}_{i}-\boldsymbol{X}^{j}\right) h^{2}
$$

The equation system for the virtual boundary flux is now formed, which can be further rewritten in the following matrix form as

$$
A X=B
$$

with $\boldsymbol{A}, \boldsymbol{X}$ and $\boldsymbol{B}$ defined as

$$
\begin{aligned}
\boldsymbol{A} & =h^{2}\left[\begin{array}{cccc}
D_{11} \Delta s_{1} & D_{12} \Delta s_{1} & \ldots & D_{1 N} \Delta s_{1} \\
D_{21} \Delta s_{2} & D_{22} \Delta s_{2} & \ldots & D_{2 N} \Delta s_{2} \\
\vdots & \vdots & \ddots & \vdots \\
D_{M 1} \Delta s_{M} & D_{M 2} \Delta s_{M} & \ldots & D_{M N} \Delta s_{M}
\end{array}\right]\left[\begin{array}{cccc}
D_{11} & D_{12} & \ldots & D_{1 M} \\
D_{21} & D_{22} & \ldots & D_{2 M} \\
\vdots & \vdots & \ddots & \vdots \\
D_{N 1} & D_{N 2} & \ldots & D_{N M}
\end{array}\right] \\
\boldsymbol{B} & =\left[\begin{array}{c}
\mathbf{U}_{\mathbf{1}} \\
\mathbf{U}_{\mathbf{2}} \\
\vdots \\
\mathbf{U}_{\boldsymbol{M}}
\end{array}\right]-h^{2}\left[\begin{array}{cccc}
D_{11} & D_{21} & \ldots & D_{N 1} \\
D_{12} & D_{22} & \ldots & D_{N 2} \\
\vdots & \vdots & \ddots & \vdots \\
D_{1 M} & D_{2 M} & \ldots & D_{N M}
\end{array}\right]\left[\begin{array}{c}
\widetilde{\boldsymbol{u}}_{1} \\
\widetilde{\boldsymbol{u}}_{\mathbf{2}} \\
\vdots \\
\widetilde{\boldsymbol{u}}_{\boldsymbol{M}}
\end{array}\right] \\
\boldsymbol{X} & =\left[\begin{array}{c}
\delta \mathbf{U}_{\mathbf{1}} \\
\delta \mathbf{U}_{\mathbf{2}} \\
\vdots \\
\delta \mathbf{U}_{\boldsymbol{M}}
\end{array}\right]
\end{aligned}
$$

In the velocity correction procedure described above, the velocity correction $\delta \boldsymbol{u}^{*}$ is computed implicitly such that the velocity $\boldsymbol{U}(\boldsymbol{X}(s), t)$ at the Lagrangian points, interpolated from the corrected velocity field $\boldsymbol{u}^{n+1}$, equals to the prescribed velocity on the immersed boundary.

Once the velocity correction is obtained, the corrected vorticity is calculated following Eq. (14) using the spatial derivatives, computed based on the DC PSE scheme [26, 27]. The algorithmic procedure of the proposed scheme can be summarized below. To update solution from time instance $(n)$ to instance $(n+1)$, we follow these steps:

1. Compute the tentative vorticity $\widetilde{\omega}$ and stream function $\widetilde{\psi}$ by solving Eqs. (7) and (8) $\left(\boldsymbol{u}^{n}\right.$ and $\omega^{n}$ are initial values)

2. Compute the tentative velocity $\widetilde{\boldsymbol{u}}$ by using $\tilde{\psi}$ to Eqs. (9)-(10)

3. Compute the matrix $\boldsymbol{A}$

4. Calculate the virtual boundary fluxes $\delta \boldsymbol{U}^{i}$ at the Lagrangian points and then compute velocity correction $\delta \boldsymbol{u}^{*}$ using Eq. (18)

5. Compute the corrected/updated velocity $\boldsymbol{u}^{(n+1)}$ at the Eulerian points using Eq. (11)

6. Use $\delta \omega^{*}=\frac{\partial \delta v}{\partial x}-\frac{\partial \delta u}{\partial y}$ to calculate the vorticity correction $\delta \omega^{*}$

7. Correct the vorticity at Eulerian points using Eq. (12)

8. Use the corrected vorticity and velocity as the initial conditions, and repeat steps 1 to 7 for the computation of the next time level. The process continues until a converged solution is achieved (steady case) or the given time is reached (unsteady case). 


\subsection{Discretization Corrected Particle Strength Exchange (DC PSE) method}

The DC PSE method originated as a Lagrangian particle-based numerical method [26], and is based on Particle Strength Exchange (PSE) operators [29, 30]. To solve partial differential equations (PDEs) using the DC PSE meshless method, Bourantas et al. [27] have reformulated the Lagrangian based DC PSE method to work in the Eulerian framework.

The Discretization Corrected PSE (DC PSE) operators were introduced to reduce the discretization error $\epsilon_{h}(x)$ in the PSE operator approximation [29, 30]. In DC PSE approximation we define a kernel function which minimizes the difference between this specific discrete operator and the actual derivative. To achieve this, we use the following expression $[26,27]$ for the derivative approximation:

$$
Q_{h}^{\boldsymbol{\beta}} f(\boldsymbol{x})=\frac{(-1)^{|\boldsymbol{\beta}|}}{\boldsymbol{\beta} !} Z_{h}^{\boldsymbol{\beta}} D^{\boldsymbol{\beta}} f(\boldsymbol{x})+\sum_{\substack{|\boldsymbol{a}|=1 \\ \boldsymbol{a} \neq \boldsymbol{\beta}}}^{\infty} \frac{(-1)^{|\boldsymbol{a}|}}{\boldsymbol{a} !} \varepsilon^{|\boldsymbol{a}|-|\boldsymbol{\beta}|} Z_{h}^{\boldsymbol{a}} D^{\boldsymbol{a}} f(\boldsymbol{x})+r_{0}
$$

with

$$
r_{0}=\left\{\begin{array}{cc}
0, & |\boldsymbol{\beta}| \text { even } \\
2 e^{-|\boldsymbol{\beta}|} Z_{h}^{0} f(\boldsymbol{x}) & |\boldsymbol{\beta}| \text { odd }
\end{array}\right.
$$

and the discrete moments defined as

$$
Z_{h}^{a}=\frac{1}{\varepsilon^{d}} \sum_{p \in N(x)}\left(\frac{x-x_{p}}{\varepsilon}\right)^{a} \eta^{\beta}\left(\frac{x-x_{p}}{\varepsilon}\right)
$$

The set of moment conditions becomes

$$
Z_{h}^{a}=\left\{\begin{array}{cc}
(-1)^{|\boldsymbol{\beta}|} \boldsymbol{\beta} ! & \boldsymbol{a}=\boldsymbol{\beta} \\
0 & \boldsymbol{a} \neq \boldsymbol{\beta} \\
<\infty & \text { otherwise }
\end{array} \quad a_{\min } \leq|\boldsymbol{a}| \leq|\boldsymbol{\beta}|+r-1\right.
$$

with $\boldsymbol{\beta}$ is a non-negative integer defined as $\boldsymbol{\beta}=\left(\beta_{1}, \beta_{2}, \ldots, \beta_{n}\right)$, where $\beta_{i}, i=1,2, \ldots, n$. The partial differential operator $D^{\boldsymbol{\beta}}$ is expressed as $D^{\boldsymbol{\beta}}=\frac{\partial^{|\beta|}}{\partial x_{1}^{\beta_{1}} \partial x_{2}^{\beta_{2}} \ldots \partial x_{n}^{\beta_{n}}}$. The kernel $\eta^{\boldsymbol{\beta}}$ is chosen as:

$$
\eta^{\boldsymbol{\beta}}(\boldsymbol{x}, \mathbf{z})=\left(\sum_{|\gamma|=a_{\min }}^{|\boldsymbol{\beta}|+r-1} a_{\boldsymbol{\gamma}}(\boldsymbol{x}) \mathbf{z}^{\gamma}\right) e^{-|\boldsymbol{z}|^{2}}=P(\boldsymbol{x}, \mathbf{z}) W(\mathbf{z}), \quad \boldsymbol{z}=\frac{\boldsymbol{x}-\boldsymbol{x}_{p}}{\varepsilon}
$$

The kernel function consists of a polynomial correction function $P(\boldsymbol{x}, \mathbf{z})$ and the weight function $W(\mathbf{z})$. Different choices for the weight function applied can be found in [31]. The unknown coefficients $a_{\gamma}(\boldsymbol{x})$ are obtained by solving the following linear system of equations $[26,27]$ :

$$
\sum_{|\boldsymbol{\gamma}|=a_{\min }}^{|\boldsymbol{\beta}|+r-1} a_{\gamma}(\boldsymbol{x}) w_{\boldsymbol{a}}^{\gamma}(\boldsymbol{x})=\left\{\begin{array}{cc}
(-1)^{|\boldsymbol{\beta}|} \boldsymbol{\beta} ! & \boldsymbol{a}=\boldsymbol{\beta} \\
0 & \boldsymbol{a} \neq \boldsymbol{\beta}
\end{array} \quad, \forall a_{\min } \leq|\boldsymbol{a}| \leq|\boldsymbol{\beta}|+r-1\right.
$$

with weights 


$$
w_{\boldsymbol{a}}^{\gamma}(\boldsymbol{x})=\frac{1}{\varepsilon^{|\boldsymbol{a}+\gamma|+d}} \sum_{p \in N(\boldsymbol{x})}\left(\boldsymbol{x}-\boldsymbol{x}_{p}\right)^{\boldsymbol{a}+\gamma} e^{-\left(\frac{\left|\boldsymbol{x}-\boldsymbol{x}_{p}\right|}{\varepsilon}\right)^{2}}
$$

To compute the approximated derivative $Q_{h}^{\boldsymbol{\beta}} f(\boldsymbol{x})$ at node $\boldsymbol{x}_{p}$, the coefficients are found by solving the above linear system of equations for $\boldsymbol{x}=\boldsymbol{x}_{p}$. Given our choice of kernel function, the DC PSE derivative approximation becomes:

$$
Q_{h}^{\boldsymbol{\beta}} f\left(\boldsymbol{x}_{p}\right)=\frac{1}{\varepsilon\left(\boldsymbol{x}_{p}\right)^{\boldsymbol{\beta}}} \sum_{\boldsymbol{x}_{q} \in \mathcal{N}\left(\boldsymbol{x}_{p}\right)}\left(f\left(\boldsymbol{x}_{q}\right) \mp f\left(\boldsymbol{x}_{p}\right)\right) \boldsymbol{p}\left(\frac{\boldsymbol{x}-\boldsymbol{x}_{p}}{\varepsilon\left(\boldsymbol{x}_{p}\right)}\right) \boldsymbol{a}^{T}\left(\boldsymbol{x}_{p}\right) e^{-\left(\frac{\left|\boldsymbol{x}_{p}-\boldsymbol{x}_{q}\right|}{\varepsilon\left(\boldsymbol{x}_{p}\right)}\right)^{2}}
$$

where $\mathbf{p}(\boldsymbol{x})=\left[p_{1}(\boldsymbol{x}), p_{2}(\boldsymbol{x}), \ldots, p_{m}(x)\right]$, with $m$ being the number of monomial used $(m=6$ and 10 for second order monomials in two dimensions) and, $\boldsymbol{a}(\mathrm{x})$ are the vectors of terms in the monomial basis and their coefficients, respectively. By using the DC PSE method, the spatial derivatives $\boldsymbol{Q}^{\beta}$ up to second order are given as:

$\boldsymbol{Q}^{1,0} \equiv \frac{\partial}{\partial x}, \quad \boldsymbol{Q}^{0,1} \equiv \frac{\partial}{\partial y}$

and

$\boldsymbol{Q}^{1,1} \equiv \frac{\partial^{2}}{\partial x \partial y}=\frac{\partial^{2}}{\partial y \partial x}, \quad \boldsymbol{Q}^{2,0} \equiv \frac{\partial^{2}}{\partial x^{2}}, \quad \boldsymbol{Q}^{0,2} \equiv \frac{\partial^{2}}{\partial y^{2}}$

\subsection{Algorithm and code verification}

We consider two benchmark problems to verify the accuracy of the numerical approach described in the previous section: the in-line oscillating cylinder in a fluid at rest and the movement of two cylinders towards each other in a fluid at rest. We note that the proposed time marching scheme is explicit. As such, in order to obtain time accurate numerical results, one needs to compute the critical time step. Details on the computation of the critical time step that ensures both stability and accuracy for the time integration scheme, as implemented in this effort, can be found in [23].

\subsubsection{Case of in-line oscillating cylinder in a fluid at rest}

To validate the proposed scheme on moving boundary flow problems, we consider the flow over an inline oscillating circular cylinder in a fluid at rest, which has been investigated both experimentally [32] and numerically [33-35]. The geometry and boundary conditions are indicated in Fig. 2. 


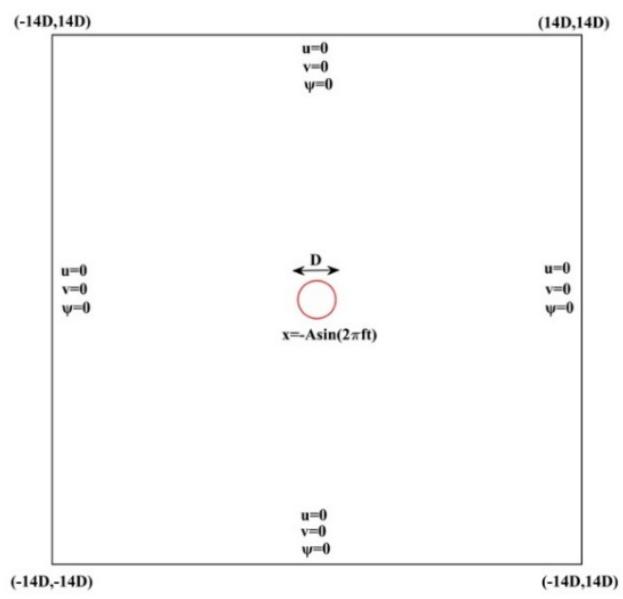

Fig. 2. A schematic of the oscillating cylinder in a fluid at rest.

The translational motion of the cylinder is given by the harmonic oscillation

$x(t)=A \sin (2 \pi f t)=-\frac{5}{2 \pi} \sin \left(\frac{2 \pi}{5} t\right)$

with $\boldsymbol{x}(t)$ being the location of the cylinder center in the $x$ - direction (oscillation direction). The oscillating amplitude is $A=\frac{5}{2 \pi}$ and the frequency $f$ of the motion is $f=\frac{1}{5}$. The parameters that characterize the flow are the Reynolds number $\operatorname{Re}=\rho U_{\max } D / \mu$, with $U_{\max }$ being the maximum velocity of the cylinder whose diameter is $\mathrm{D}$, and the Keulegan-Carpenter number $K C=U_{\max } / f D$, which is the inverse of the Strouhal number. In the present study, $R e$ is set equal to 100 and the parameter $K C$ is set equal to 5 , corresponding to the experiments and the numerical simulations of Dutsch et. al [32]. For $R e=100$ and $K C=5$ the flow remains two-dimensional with periodic vortex shedding.

The size of the computational domain is set to $14 D \times 14 D$ (as shown in Fig. 2), with the cylinder located initially at the center. We apply no-slip boundary conditions to the boundaries of the flow domain. Successively finer nodal distributions were considered; starting with a grid spacing of $h_{1}=0.1, h_{2}=0.05$, $h_{3}=0.025$ and $h_{4}=0.0167$, which results in 19,881, 78,961, 314,721 and 707,281 nodes, respectively. We use denser grids (or nodal distributions) to examine the sensitivity of the numerical solution to grid resolution, and to ensure a grid independent numerical solution. The cylinder was initially at rest and progressed in time until periodic vortex shedding was established. The mesh with resolution $h=0.025$ or 314,721 nodes provided a grid independent numerical solution with the results from the two finest grids almost indistinguishable. The time step used in the simulation is set to $d t=5 \times 10^{-3}$. The cylinder is represented by 360 points due to boundary segment length $d s \cong h$. The prescribed velocity boundary conditions (Eq. (36)) at the surface of the moving cylinder are enforced using our scheme described above. At each time step the velocity of the cylinder is given by:

$$
\boldsymbol{u}(t)=-\cos \left(\frac{2 \pi}{5} t\right)
$$



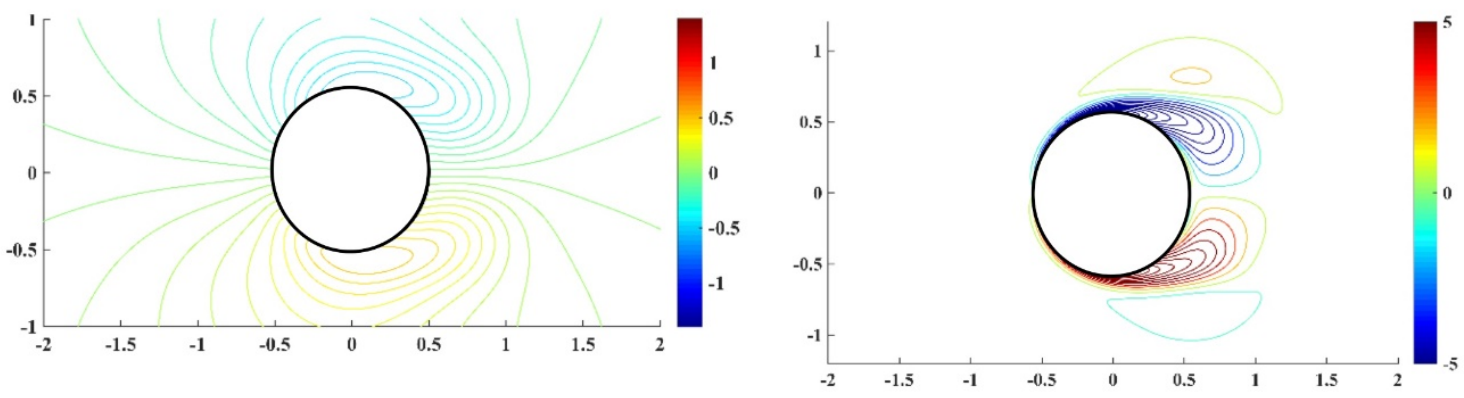

(a)
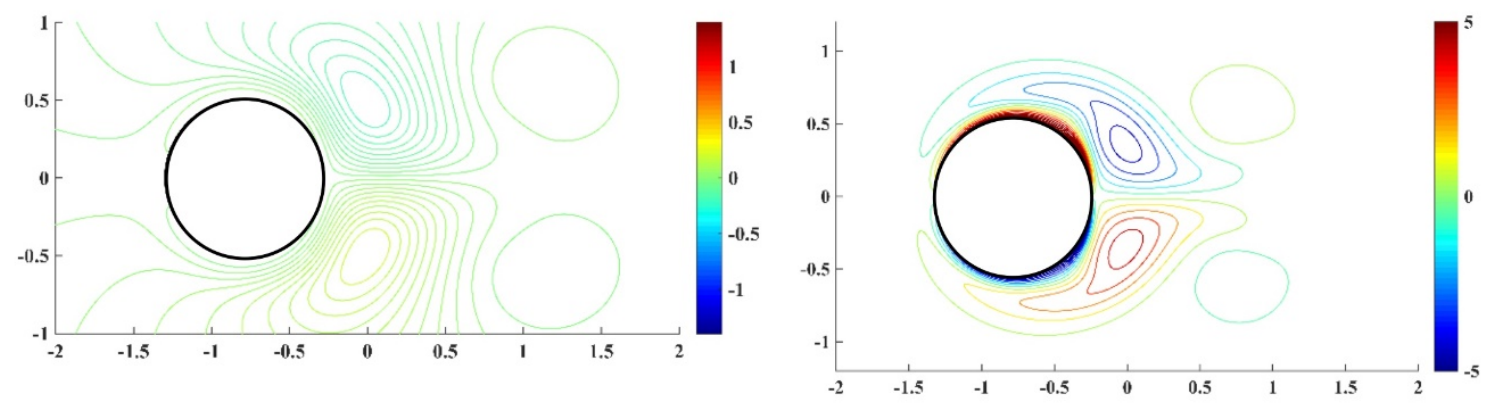

(b)
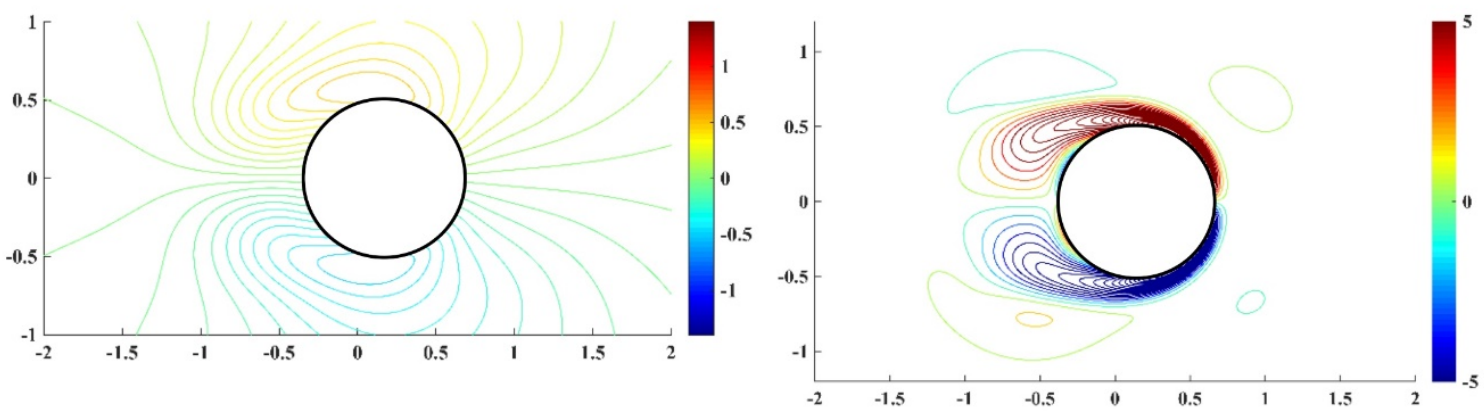

(c)
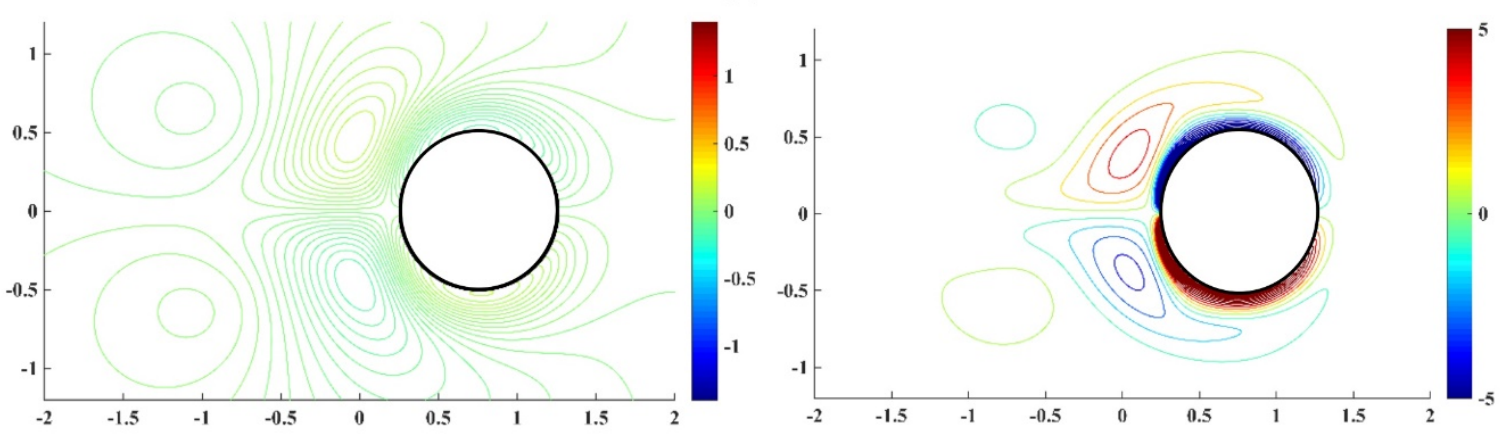

(d)

Fig. 3. Instantaneous streamlines (left frame) and instantaneous vorticity contours (right frame) of in-line oscillations of a circular cylinder at four different phase angle positions: (a) $0^{\circ}$ (b) $96^{\circ}$ (c) $192^{\circ}$ and (d) $288^{\circ}$ at $R e=100$ and $\mathrm{KC}=5$. These results are in good agreement with those reported in [33, 37]. 

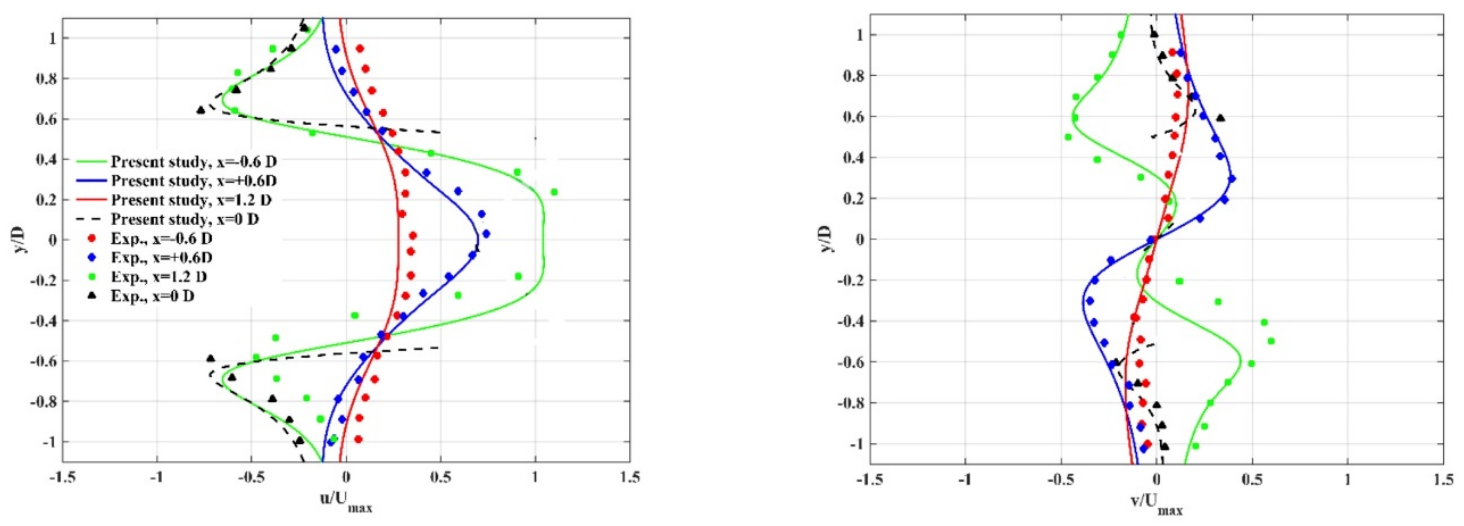

(a) $180^{\circ}$
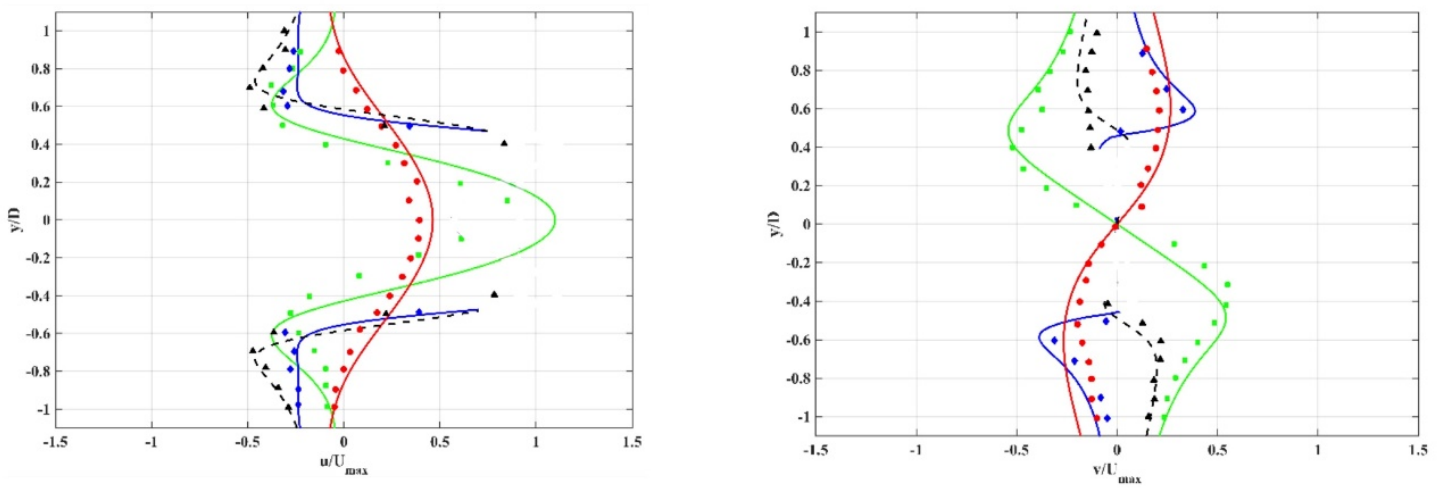

(b) $210^{\circ}$
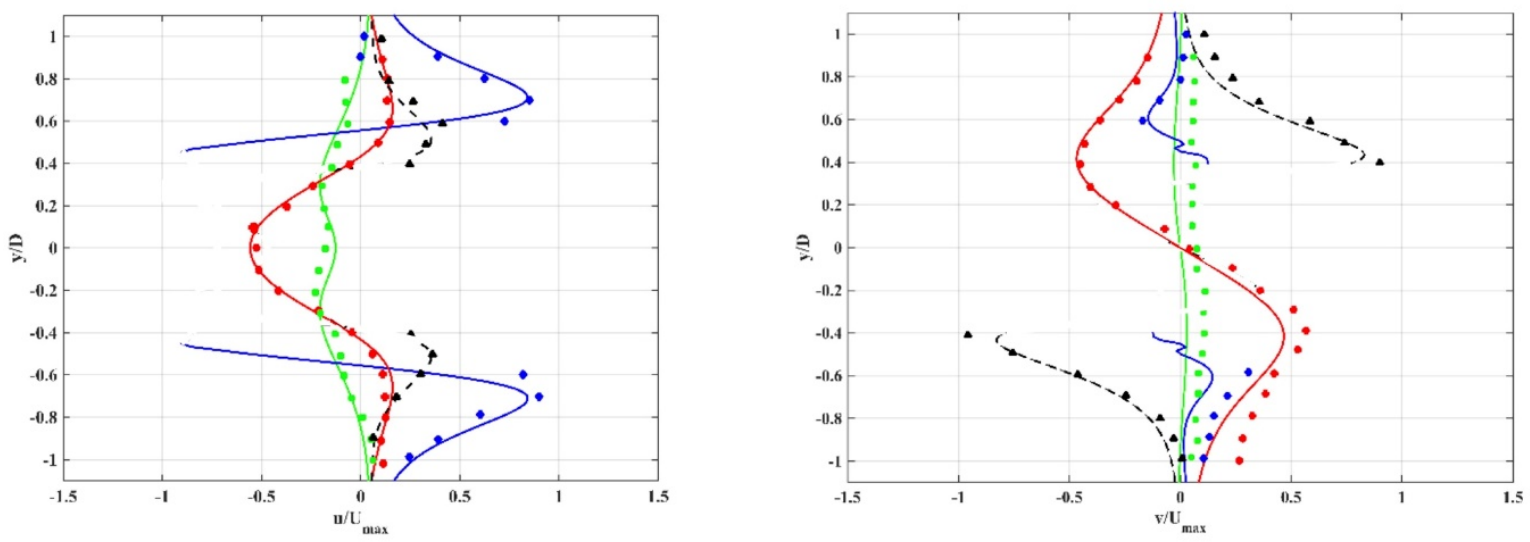

(c) $330^{\circ}$

Fig. 4. Comparison of the velocity profiles for $R e=100, K C=5$ between the present study and experimental results (dots) of Dütsch et al. [32] for different phase angle positions: (a) $2 \pi f t=180^{\circ}$ (b) $2 \pi f t=210^{\circ}$ and (c) $2 \pi f t=330^{\circ}$.

Fig. 3 displays the stream function and vorticity contours at four different phase-angles obtained at different instants $\left(0^{\circ}, 96^{\circ}, 192^{\circ}\right.$, and $\left.288^{\circ}\right)$. Two fixed stagnation points (vortices) at both ends of the cylinder are developed. As the cylinder moves to the left, two thin boundary layers develop on the upper and lower sides (see Fig. 3), which, as time evolves, separate and form two counter-rotating vortices of same strength. 
Vortices stop forming, as the body reaches its extreme left location, as shown in Fig. 3(b). Then, as the cylinder moves backwards (to the right direction), the same process takes place on its right side. The two vortices generated earlier, split and move further away from the cylinder as shown in Fig. 3(c). The results obtained using the proposed scheme, are in good agreement with the corresponding results reported in [32, 36], highlighting that the present method captures the dynamics of the vorticity field. We validate the scheme by quantitatively comparing our numerical findings with the experimental results reported in [32, 36]. Fig. 4 shows a comparison of the computed velocity profiles at four locations in the $x$ - axis and three different phase angles with the experimental data. A good agreement between the two set of data is obtained.

\subsubsection{Case of two cylinders moving towards each other in a fluid at rest}

As a second example, we examine the flow of two cylinders approaching each other in a fluid at rest. We consider successively denser uniform Cartesian grids, with grid spacing $h=0.1,0.05$ and 0.025 (51,681, 205,761 and 821,121 number of nodes, respectively) to ensure a grid independent numerical solution. A mesh with resolution of $h=0.05$ provided a grid independent numerical solution (the results on the two finest grids are almost indistinguishable). The spatial domain and boundary conditions are shown in Fig. 5.

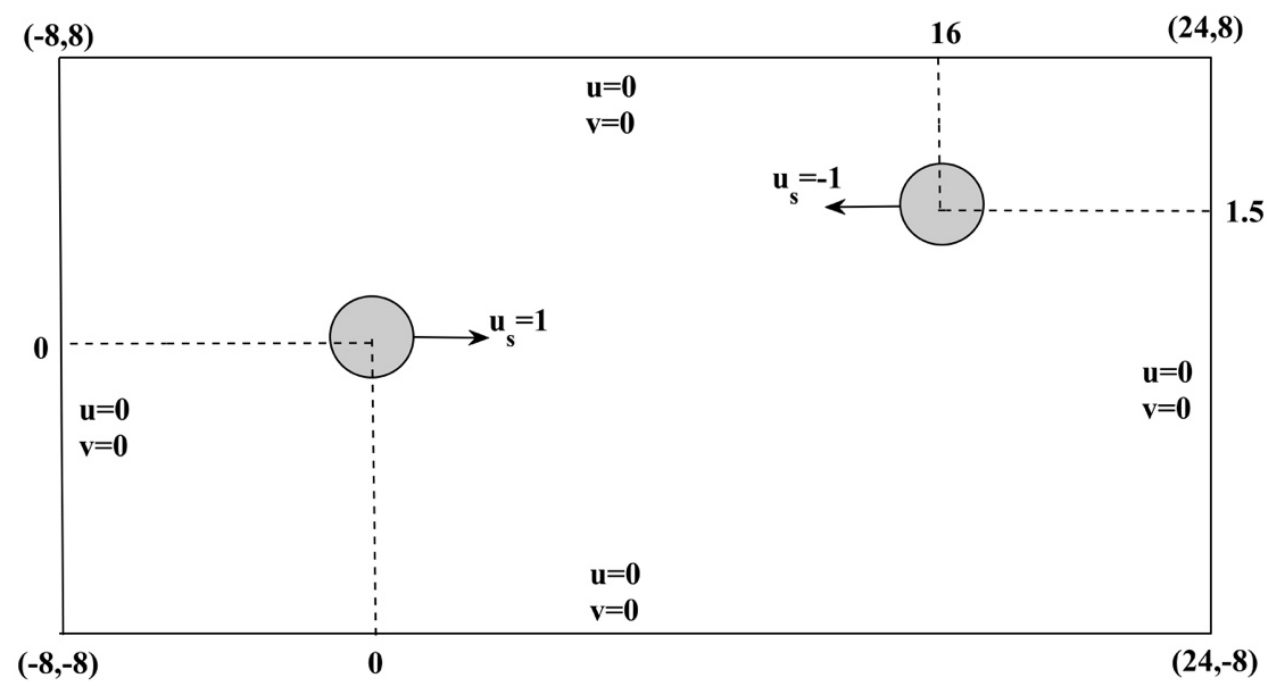

Fig. 5. Geometry and flow boundary conditions for the two moving cylinders.

For Reynolds number $R e=40$, the critical time step computed using Gershgorin theorem is $d t_{\text {critical }}=$ 0.014. The time step used in the simulation is set to $d t=10^{-3}$. The updated position of the lower and upper cylinders are given through the translational motion of their centers $\left(x_{l}, y_{l}\right)$ and $\left(x_{u}, y_{u}\right)$ given by:

$x_{l}=\left\{\begin{array}{cc}\frac{4}{\pi} \sin \left(\frac{\pi t}{4}\right), & 0 \leq t \leq 16 \\ t-16 & 16 \leq t \leq 32\end{array} \quad\right.$ and $y_{l}=0$

and

$x_{u}=\left\{\begin{array}{cc}16-\frac{4}{\pi} \sin \left(\frac{\pi t}{4}\right), & 0 \leq t \leq 16 \\ 32-t & 16 \leq t \leq 32\end{array}\right.$ and $y_{l}=1.5$ 


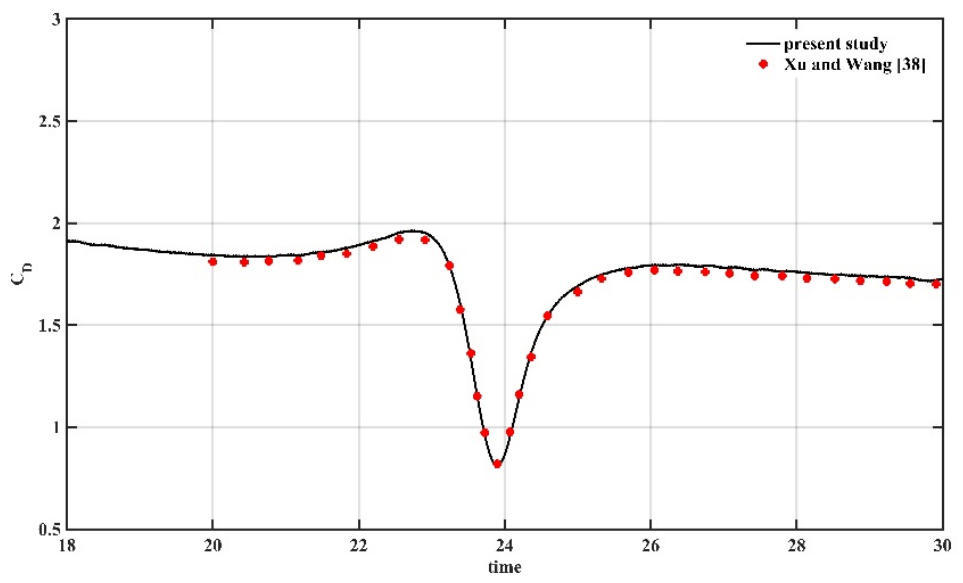

(a)

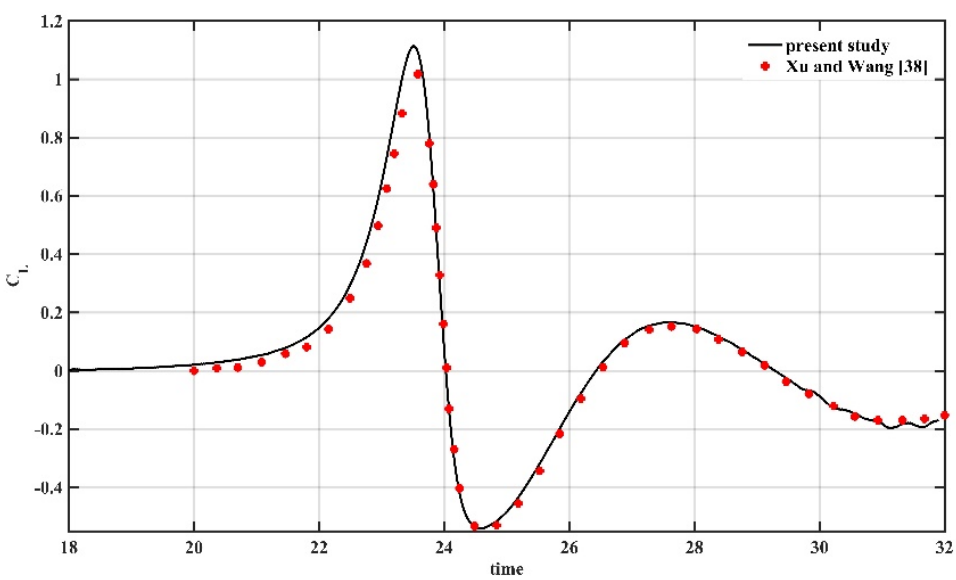

(b)

Fig. 6. Time evolution of drag $\left(C_{D}\right)$ and lift $\left(C_{L}\right)$ coefficients for the upper cylinder (case of two cylinders moving towards each other). Simulation results are compared with reported results from the simulations by $\mathrm{Xu}$ and Wang [38].

Both cylinders oscillate about their initial positions, and then move toward each other at time $t=16$. Fig. 6 displays the time evolution of drag and lift coefficients for the upper cylinder. As seen in Fig. 6, the numerical results obtained by the proposed scheme are in a very good agreement with those reported in [38]. The drag slightly increases when the two cylinders approach to each other, and decreases when they are close to each other. On the other hand, the two cylinders are repulsive when approaching each other and then become attractive after passing each other.

\section{Hydrodynamic Performance of Fish-like Locomotion}

We implement the computational approach described above to simulate fish-like locomotion and investigate the impact of the undulatory motion of the body and the flapping of a caudal fin on the generation of the hydrodynamic loads: that is, the lateral side force and thrust or negative drag. Particularly, we identify the kinematics parameters most affecting the performance of fish-like swimming. 
In our study, we model the motion of the fish during swimming, and we identify characteristics of this complex movement. A Newtonian fluid is assumed, and the flow equations are the incompressible NavierStokes equations. The fish performs an undulatory motion of its body as described in Section 3.1 [38-40]. Fig. 7 displays the flow domain along with the applied boundary conditions. The dimensions of the flow domain along the stream-wise and lateral directions are $30 L$ and $20 L$, respectively, with $L$ being the fish body length $(L=1)$. The fish is located at a distance of $10 L$ from the in-flow boundary. We employ Dirichlet conditions for the velocity field on the inlet, upper and lower walls $\left(U_{\infty}=1, v=0\right)$, and Neumann conditions at the outlet. We obtain the non-dimensional formulation of the governing equations by using the fish body-length $L(L=1)$. The Reynolds number is defined as $R e=L U_{\infty} / v_{f}$, where $v_{f}$ is the kinematic viscosity of the fluid.

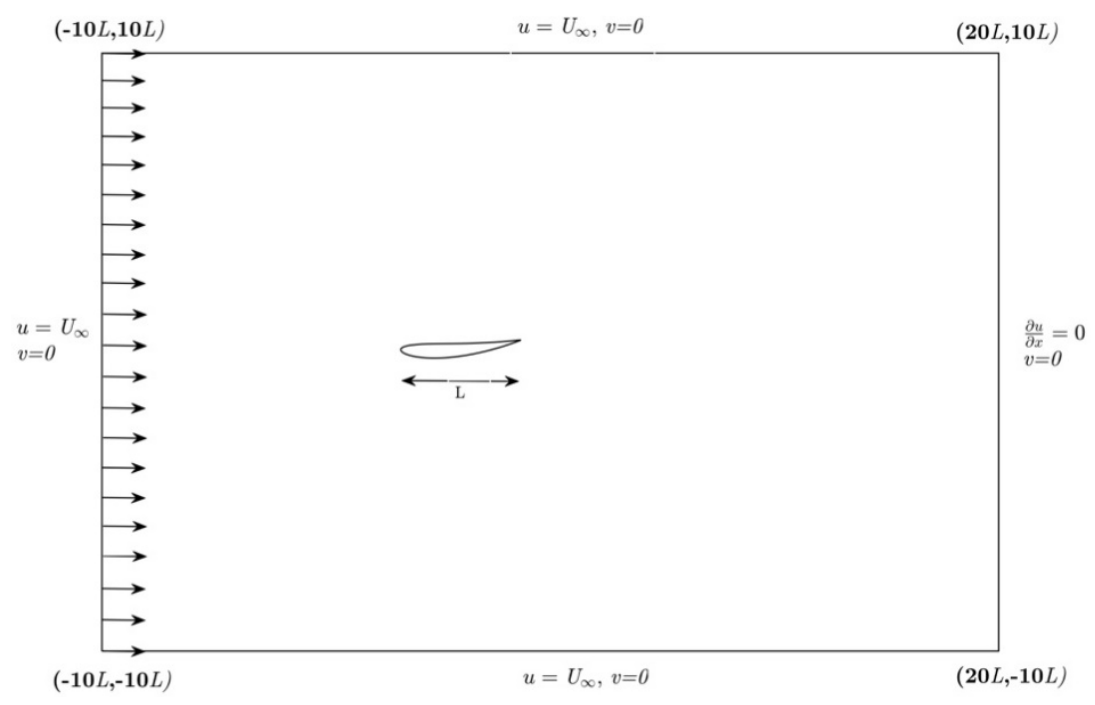

Fig. 7. Flow domain and corresponding boundary conditions.

\subsection{Sensitivity analysis}

For the sake of comparison with previously-published studies [14], the symmetric NACA 0012 airfoil is considered as the fish body in absence of the undulatory motion. The chord of the airfoil is taken as the fish-backbone which is assumed to undergo lateral deformation according to the following wave equation [14]:

$$
h(x, t)=\left(c_{0}+c_{1}\left(\frac{x}{L}\right)+c_{2}\left(\frac{x}{L}\right)^{2}\right) \cos \left(2 \pi k \frac{x}{L}-2 \pi f t\right) \quad 0 \leq \frac{x}{L} \leq 1
$$

where $x$ is the position along the streamwise direction, $L$ is the length of the fish. The coefficients $c_{0}, c_{1}$, and $c_{2}$ are set equal to $0.02,-0.0825$, and 0.1625 , respectively, to simulate the kinematics of a steadily swimming saithe fish [14]. Given the aforementioned numerical values of the coefficients $c_{i}$, the maximum lateral amplitude of the tail oscillation $\hat{h}_{\text {tail }}$ is set equal to $0.1 L . k$ is the wave number and $f$ denotes the frequency of the lateral oscillations. Hereafter, we use the normalized lateral amplitude of the tail denoted by $h_{\text {tail }}=\hat{h}_{\text {tail }} / L$. Fig. 8 shows a sketch of the fish locomotion over one period of the undulatory motion. 

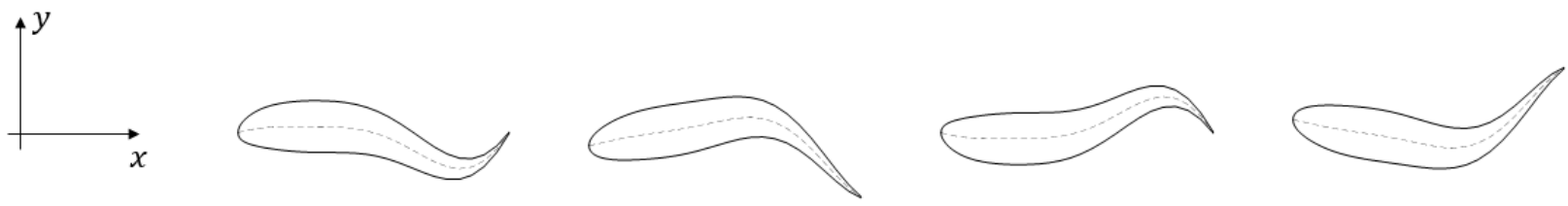

Fig. 8. Sketch of the fish locomotion.

To analyze the hydrodynamic performance of the fish swimming under different operating conditions, we introduce the dimensionless parameters, namely the Strouhal number $S_{r}$ defined as $S_{r}=\frac{2 f \widehat{h}_{\text {tail }}}{U_{\infty}}$ where $U_{\infty}$ is the freestream velocity at the inflow boundary of the simulation domain, $\hat{h}_{\text {tail }}$ and $f$ are defined above. We show in Table 1 the numerical values of the parameters used in the simulations.

Table 1. Simulation parameters used in the present study

\begin{tabular}{ccccc}
\hline Parameter & Reynolds number & Strouhal number & Wave number & Tail amplitude \\
& $R_{e}$ & $S_{r}$ & $k$ & $h_{\text {tail }}$ \\
Value range & 500 & $0.1-0.8$ & 1 & $0.05-0.2$ \\
\hline
\end{tabular}

In implementing the simulations, we discretize the fluid domain with 858,496 nodes in total, locally refined around the immersed fish-like body. The grid spacing in the finest grid around the immersed object is $h_{\text {ref }}=0.0028125$. The time step is estimated based on Gershgorin theorem as described in [23] and is set equal to $d t=5 \times 10^{-4}$. The total simulation time was set to six times the period of undulatory motion, resulting in 12,000 time steps. The selection of the grid size and time step is made to guarantee the stability of the numerical soltuion and obtain accurate hydrodynamic force coefficients. The numerical solution is found to reach steady state after two cycles. The time-averaged and root mean square values of the hydrodynamic force coefficients are computed over four periods of the lateral oscillations.

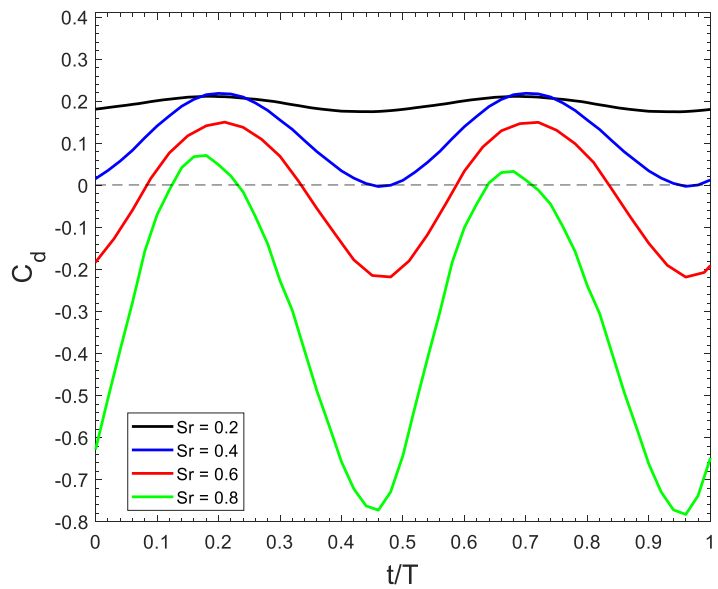

(a) Drag coefficient $C_{d}$

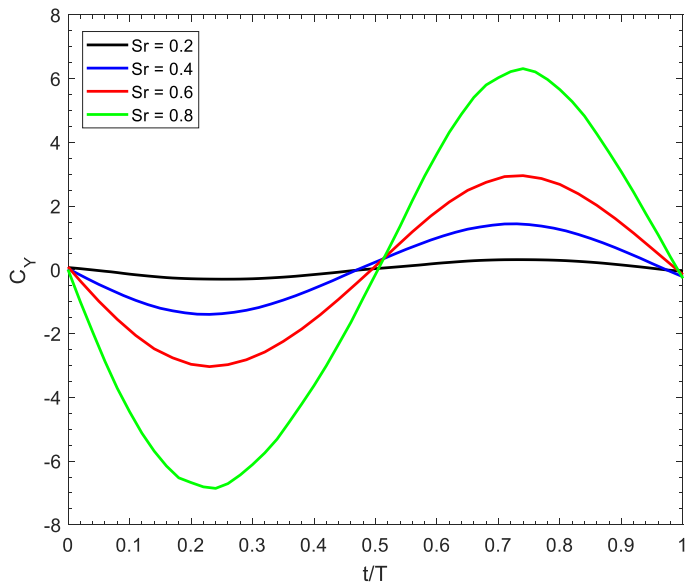

(b) Lateral side force coefficient $C_{Y}$

Fig. 9. Time histories of (a) the drag coefficient and (b) the lateral side force coefficient over one undulating cycle for varying Strouhal number $S_{r}$. 
The generation of the hydrodynamic forces and wake pattern downstream of the swimming fish when varying the Strouhal number is investigated over the range between 0.1 and 0.8 . The force on the immersed boundary is calculated using the virtual boundary flux $\delta \boldsymbol{U}$, as $\boldsymbol{F}=\frac{\rho \delta \boldsymbol{U}}{\delta t}$. Therefore, the drag $F_{D}(x$ component of the boundary force $\boldsymbol{F}$ ) and lateral side forces $F_{Y}$ ( $y$-component of the boundary force $\boldsymbol{F}$ ) are given as $F_{D}=\boldsymbol{F} \cdot \widehat{\boldsymbol{x}}$ and $F_{Y}=\boldsymbol{F} \cdot \widehat{\boldsymbol{y}}$, with $\widehat{\boldsymbol{x}}$ and $\widehat{\boldsymbol{y}}$ being the unit vectors in $x$ - and $y$-directions, respectively. The hydrodynamic coefficients denoted by $C_{d}$ and $C_{Y}$ are computed as $C_{D}=\frac{F_{D}}{\frac{1}{2} \rho U_{\infty}^{2} L}$ and $C_{Y}=\frac{F_{Y}}{\frac{1}{2} \rho U_{\infty}^{2} L}$, dividing the corresponding forces by $\frac{1}{2} \rho U_{\infty}^{2} L$. We note that the variations of $S_{r}$ is obtained by changing the value of the oscillation frequency while maintaining the maximum amplitude of the tail oscillation $h_{\text {tail }}$ equal to 0.1 . We set the Reynolds number equal to 500 for all subsequent simulations for the sake of comparison and validation of the present numerical results with those reported in the literature [14].

The undulatory motion of the fish, generates vortices which traverse downstream and start shedding from the trailing-edge of the fish. The generated vortices, rotate in clockwise or anti-clockwise direction, and form wakes. Therefore, fish experiences unsteady hydrodynamic forces (due to generated wakes) that reflect to unsteady $C_{d}$ and $C_{Y}$ hydrodynamic coefficients. The time histories of the drag and lateral sideforce coefficients over one cycle of the undulatory motion are shown in the plots of Fig. 9. The results are shown for different values of $S_{r}$ over one undulation cycle. The time is presented in dimensionless form using the period of undulatroy motion, denoted by T. Fig. 9(a) shows that the the time history of the drag coefficient $C_{d}$ shifts to lower values when increasing $S_{r}$. At some level of $S_{r}$, negative values of the drag coefficient are obtained. This indicates the production of positive thrust and then thrust-based swimming of the fish. For $S_{r} \geq 0.6$, the numerical results show the capability of the fish to generate thrust, or negative drag, over the entire undulatory cycle. As expected, the side-force coefficient $C_{Y}$ is symmetric about the $x$ axis resulting in zero average value and its amplitude increases for higher values of the Strouhal number as depicted in Fig 9(b). This indicates that the fish body needs to spend more power to ensure faster lateral deformation via its flexible backbone.

We show in Fig. 10 the power spectra of the lateral side-force coefficient and the drag coefficient. The Strouhal number is set equal to 0.4 . The $C_{Y}$ spectrum shows a peak with the highest amplitude occuring at the undulation frequency. On the other hand, the $C_{d}$ spectrum shows a dominant peak at twice the undulation frequency. We note that the mean value of the drag coefficient was subtracted when evaluating the power spectrum. These observations are consistent with the numerical results obtained by Khalid et al. [14].

Next, we consider the wake pattern resulting from the undulatory motion of the fish. The voriticy contours of the wake obtained for different $S_{r}$ is plotted in Fig. 11. When the fish body experiences wave-like deformation as illustrated in Fig. 8, the shear layer formed along the body deataches from its trailing edge and leads to the shedding of alternating vortices in the downstream direction. Setting $S_{r}=0.2$, we observe a reccuring pattern of swirling vortices of opposite signs. This pattern is similar to the well-known vonKarman vortex street observed in fluid flows over blunt bodies. As the Strouhal number is increased by increasing the frequency of the undulatory motion, the two layers of alternating vortices merge towards each other. Of interest, unlike the case of low $S_{r}$, the formed vortices with negative vorticity are placed underneath those with positive vorticity when setting $S_{r}=0.6$ and $S_{r}=0.8$. This corresponds to the reverse von Karman vortex street. The vortices dissipate after traversing a distance of about six times the body length in the wake. The simulation results of the wake structure are consistent qualitatively and quantitevily with those reported by Khalid et al. [14]. 


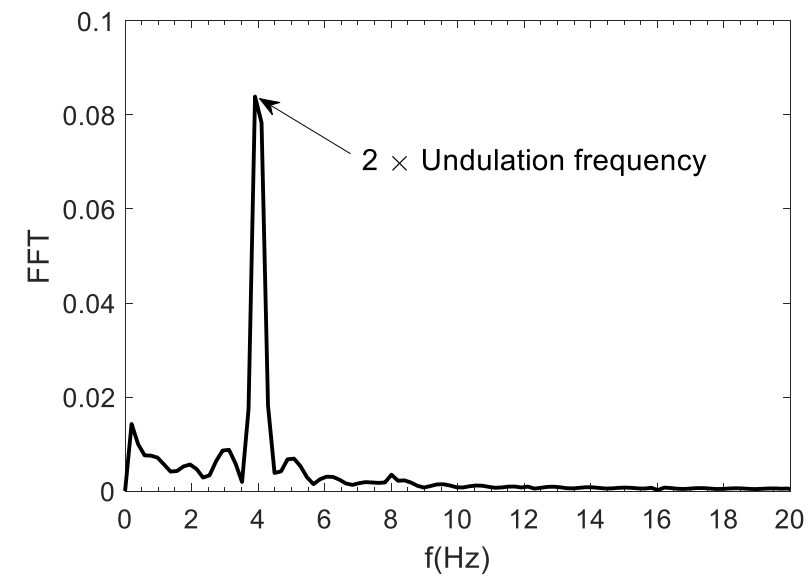

(a)

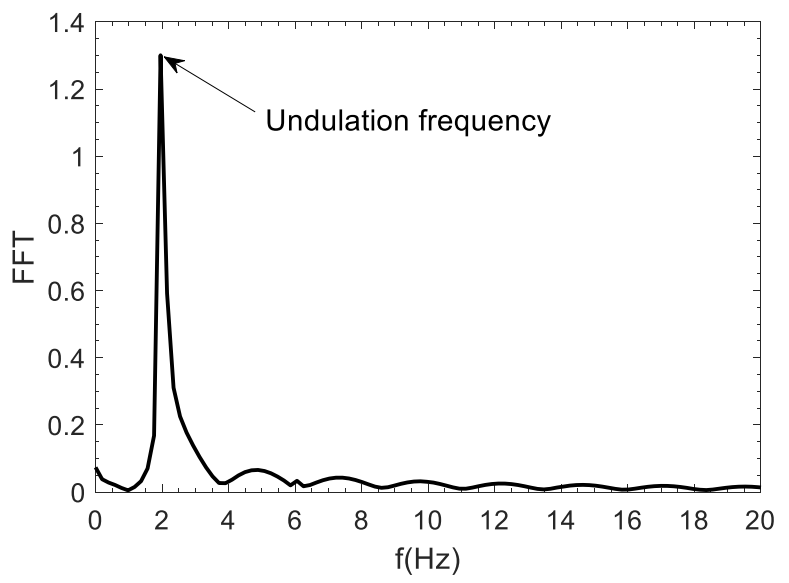

(b)

Fig. 10. Power spectra of (a) the drag coefficient and (b) the lateral side-force coefficient. The Strouhal number is equal to 0.4 .

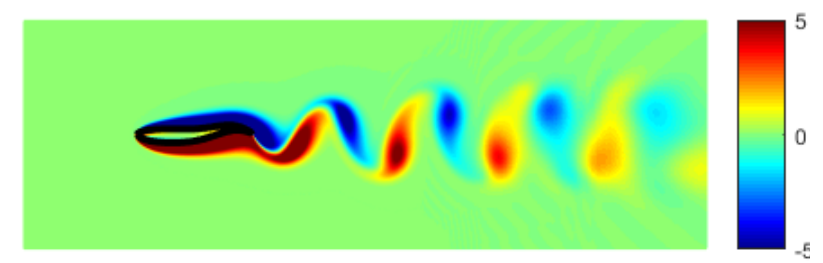

(a) $S r=0.2$

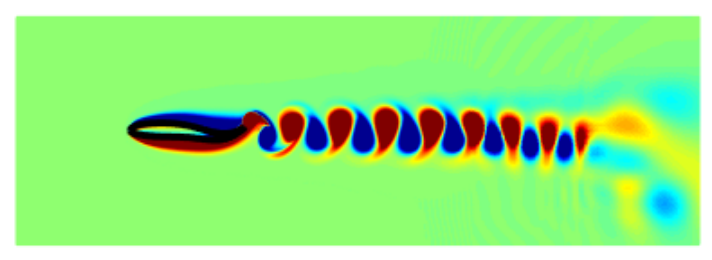

(c) $S r=0.6$

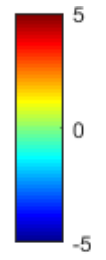

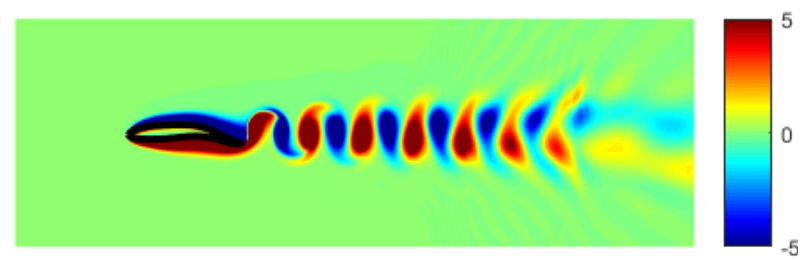

(b) $S r=0.4$

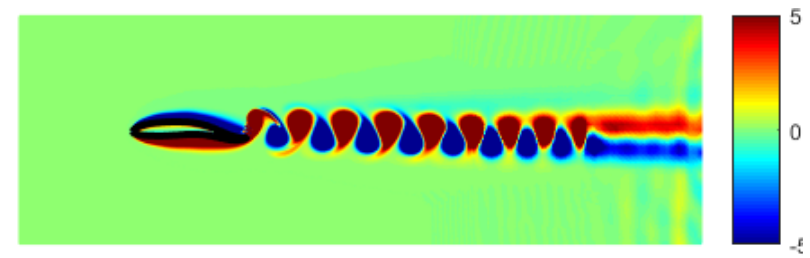

(d) $S r=0.8$

Fig. 11. Vorticity pattern of the wakes generated by the fish-like locomotion at $R_{e}=500$ for different Strouhal number. The magnitude is varying from -5 to 5 as reported in Khalid et al. [14].

To further verify the accuracy of the current numerical approach used to simulate fish-like swimming, we show in Figs. 12(a) and 12(b) the variations of the time-averaged values of the drag coefficient and RMS values of the lateral side-force coefficient, respectively, with the Strouhal number. The numerical results are compared against those obtained by Khalid et al. [14]. A good agreement between the two sets of data is observed. This demonstrates the capability of the present computational model to predict the hydrodynamic loads generated by fish-like undulatory movement. As shown in Fig. 12(a), a positive drag is obtained when setting the Strouhal number less than 0.545. Beyond this critical value, the drag shifts from positive to negative values. We note that the Strouhal number is varied by changing the value of the undulation frequency while keeping the amplitude of the tail oscillations $h_{\text {tail }}$ constant and equal to $0.1 L$. 
Fig. 12(b) shows an increasing trend of the side-force coefficient when increasing $S_{r}$. The slope of the curve increases significantly for $S_{r} \geq 0.2$.

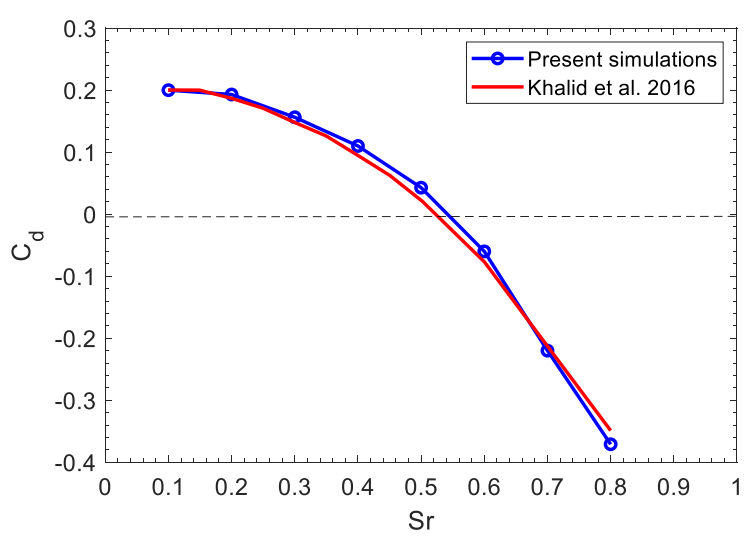

(a)

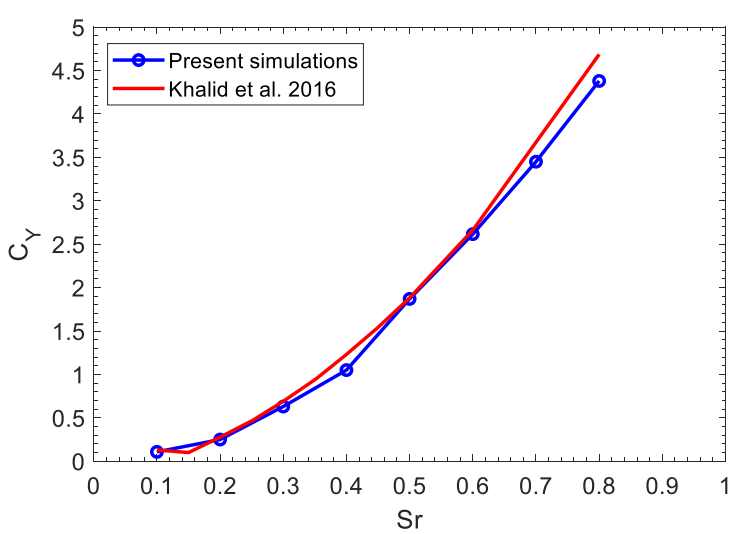

(b)

Fig. 12. Variations of (a) the time-averaged drag coefficient and (b) root-mean square (RMS) value of the lateral side force coefficient with the Strouhal number $S_{r}$. Comparison against the numerical results obtained by Khalid et al. [14].

\subsection{Effect of undulatory tail oscillations}

Next, we investigate the effect of the tail oscillation amplitude $h_{\text {tail }}$ on the hydrodynamic performance of the fish. We show in Fig. 13 the simulated undulatory motion sequence in periodic straight line swimming mode for different tail oscillation amplitudes. We note that the length of the fish-like body $L$ and the inlet velocity $U_{\infty}$ are maintained fixed. As such, the Reynolds number $R e$ remains constant and equal to 500 for all simulation cases.

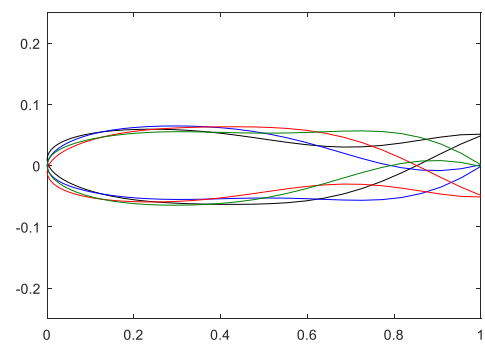

(a) $h_{\text {tail }}=0.05$

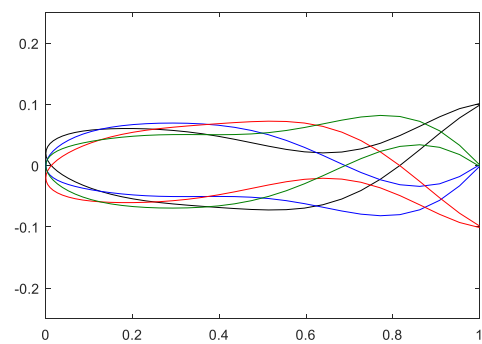

(b) $h_{\text {tail }}=0.1$

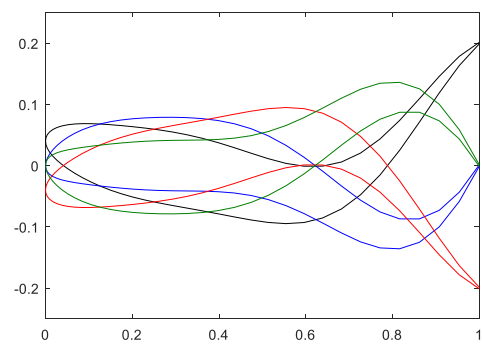

(c) $h_{\text {tail }}=0.2$

Fig. 13. Simulated undulatory motion sequence in periodic straight line swimming mode for different tail oscillation amplitudes.

In Figs. 14(a) and 13(b) we plot the variations of the time-averaged values of the drag coefficient and RMS values of the lateral side-force coefficient, respectively, with the Strouhal number while increasing the value of $h_{\text {tail }}$ from $0.05 \mathrm{~L}$ to $0.15 \mathrm{~L}$. These figures provide an insight on the combined effect of the undulation frequency and amplitude on the generation of hydrodynamic loads. In Fig. 14(a), the dashed horizontal line represents the transition from drag-based to thrust-based swimming. Its intersection with the $C_{d}$ vs. $S_{r}$ curve indicates the thrust-based propulsion Strouhal number; that is, the required amplitude of oscillations and/or undulation frequency for the fish to start producing positive thrust at the freestream velocity. The results demonstrate that fish with smaller amplitudes need to produce faster body deformation to generate positive 
thrust. If the Strouhal number is kept constant, increasing the ampitude of the tail oscillations shifts the side-force coefficient to higher values. This is not the case for the drag coefficient over the full range of the Strouhal number $S_{r}$ considered in the present study. Indeed, a lower positive drag is obtained when decreasing $h_{\text {tail }}$ for $S_{r} \leq 0.4$. Beyond this value, setting $h_{\text {tail }}$ equal to 0.1 is found to enable the highest thrust generation. This indicates that at a certain undulation frequency range, amplifying the tail oscillations does not necessarily lead to more thrust generation or higher swimming speed.

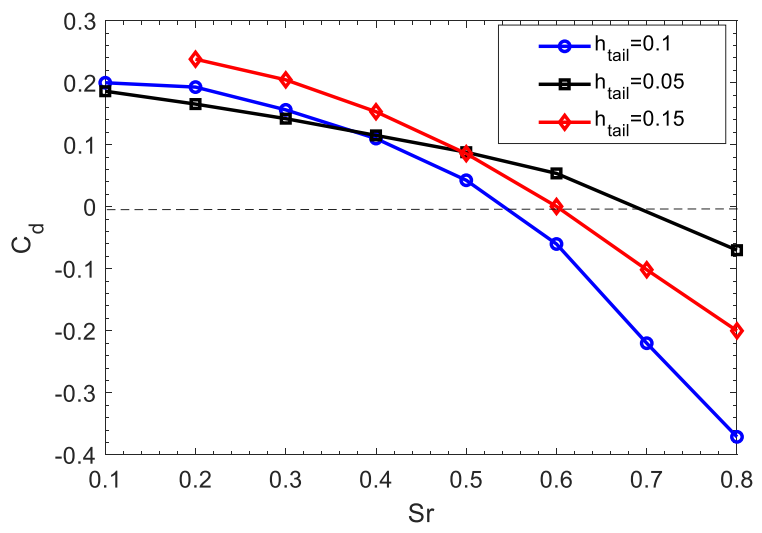

(a)

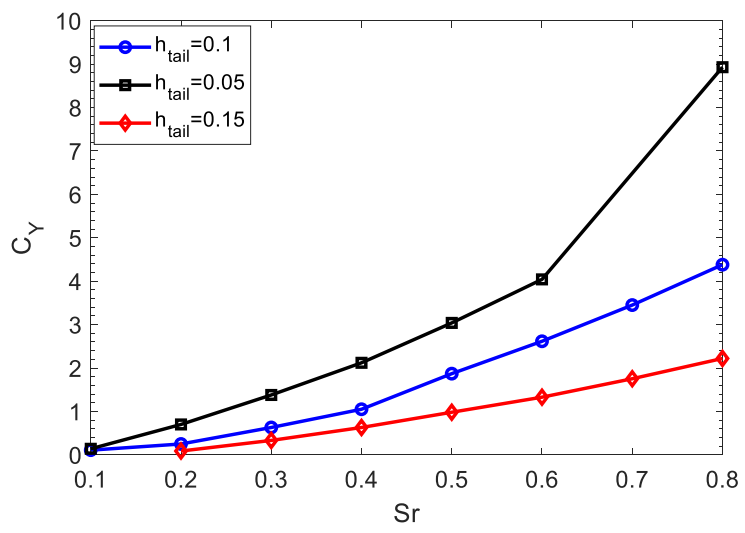

(b)

Fig. 14. Effect of the maximum lateral amplitude of the tail on (a) the drag force coefficient and (b) the lateral force coefficient. Results are obtained for varying Strouhal number values.

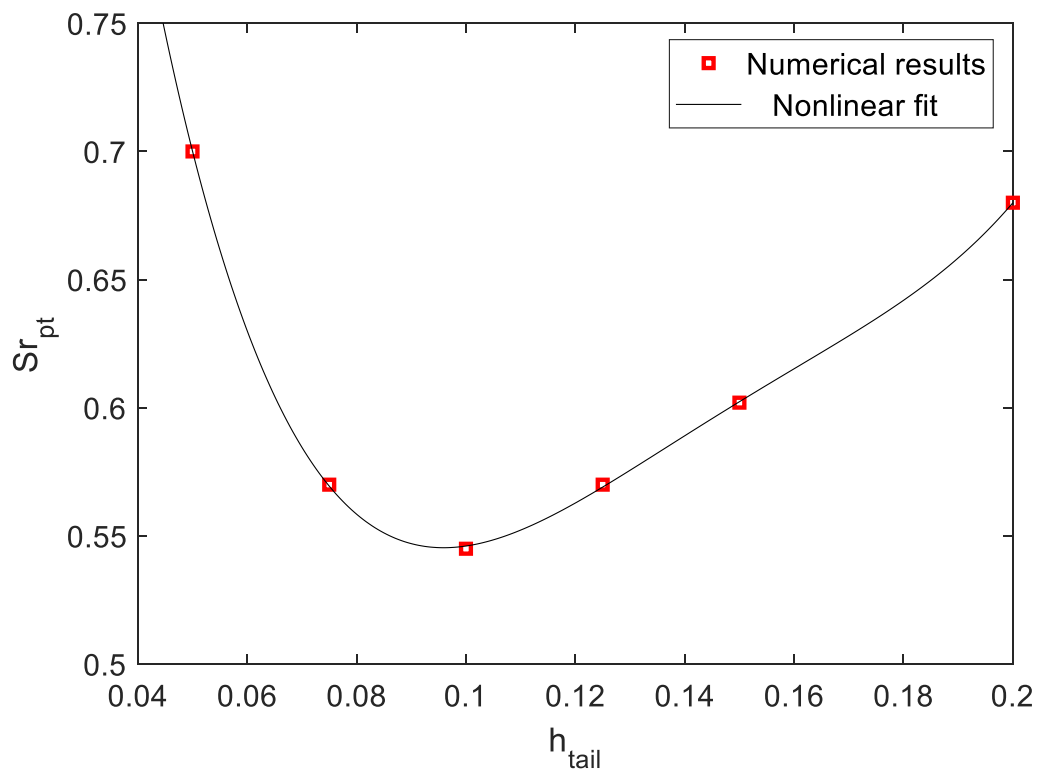

Fig. 15. Thrust-based propulsion Strouhal number as function of the maximum lateral amplitude of the tail.

In Fig. 15, we plot the variations of the thrust-based propulsion Strouhal number, denoted by $S r_{p t}$, with the normalized amplitude of the tail oscillations $h_{\text {tail }}$. This amplitude is varied from 0.05 to 0.2 . The amplitude is actually related to the size of fish. For instance, a fish with a tail oscillating at a normalized amplitude of 0.05 needs to deform its body following a wave-like motion at a frequency $f$ to reach thrust-based swimming. On the other hand, a larger fish with a tail oscillating at a normalized amplitude of 0.2 requires lower undulation frequency equal to $0.24 f$ to produce positive thrust. We observe the occurrence of local 
minimum at $h_{\text {tail }}=0.1$. Then, $S r_{p t}$ increases gradually with increasing $h_{\text {tail }}$. Fig. 15 is indicative of the kinematic requirements of the undulating body to secure positive thrust generation and forward motion. Of interest, we notice the existence of an optimal value for the lateral oscillations that minimizes the Strouhal number required to enable the undulatory body to produce positive thrust.

\subsection{Effect of flapping a caudal fin}

Next, we attach a flapping caudal fin with a symmetric NACA 0012 profile to the tail of the fish and examine its effect on the hydrodynamic performance. The flapping motion of the caudal fin is represented by the plunge and pitch components [15]

$$
\left\{\begin{array}{c}
h_{c}=[h(x, t)]_{x=L} \\
\theta_{c}=\theta_{\max } \cos \left(2 \pi f_{c} t+\varphi\right)
\end{array}\right.
$$

where $h_{c}$ and $\theta_{c}$ represent the time-varying plunge displacement and pitch angle, respectively. $\theta_{\max }$ denotes the amplitude of the pitch angle, $f_{c}$ is the pitching frequency, and $\varphi$ is the phase angle between the plunging and pitching motions. $\varphi$ represents also the phase angle between the body undulation and the flapping of the caudal fin. Fig. 16 illustrates the locomotion of the fish body equipped with a flapping caudal fin.
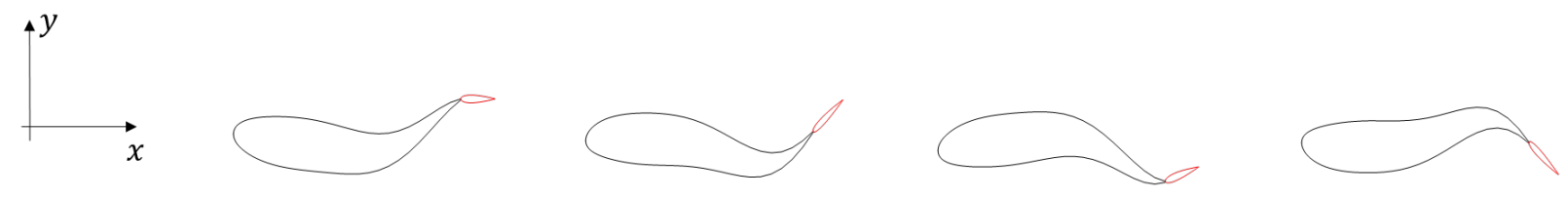

Fig. 16. Sketch of the fish locomotion with flapping caudal fin.

We show in Fig. 17 the computed drag and lateral side force coefficients as function of the Strouhal number. The simulation results are obtained with and without caudal fin while keeping the flapping frequency of the caudal fin $f_{c}$ equal to the frequency of the lateral oscillations $f$. The amplitude of the flapping angle $\theta_{\text {max }}$ is set equal to $15^{\circ}$ and the phase angle $\varphi$ is taken equal to zero. We observe a significant improvement in the thrust generation when incorporating the flapping caudal fin. For instance, the thrust increases by about $180 \%$ at $S_{r}=0.6$. Furthermore, the thrust-based propulsion occurs at a lower value of the Strouhal number when including the caudal fin. This indicates the benefit of the caudal fin movement in achieving superior swimming performance. The lateral side force coefficient $C_{Y}$ is also shifted to higher values when incorporating the caudal fin. The impact of the caudal fin on $C_{Y}$ is more pronounced as the Strouhal number is increased. 


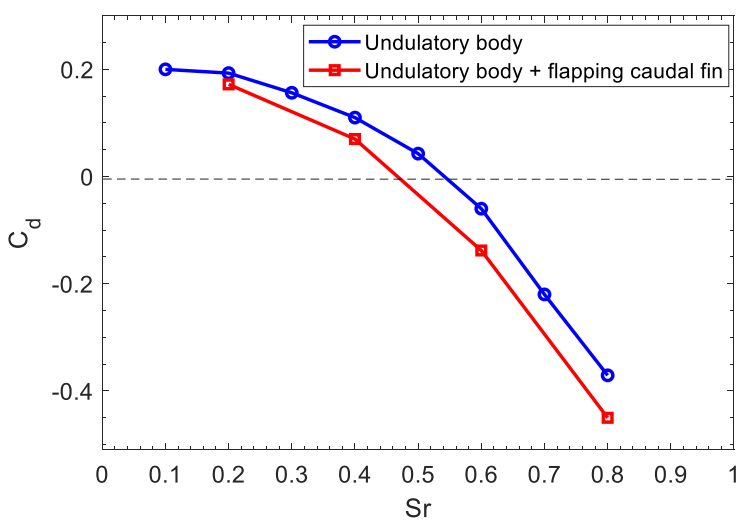

(a) Drag coefficient $C_{d}$

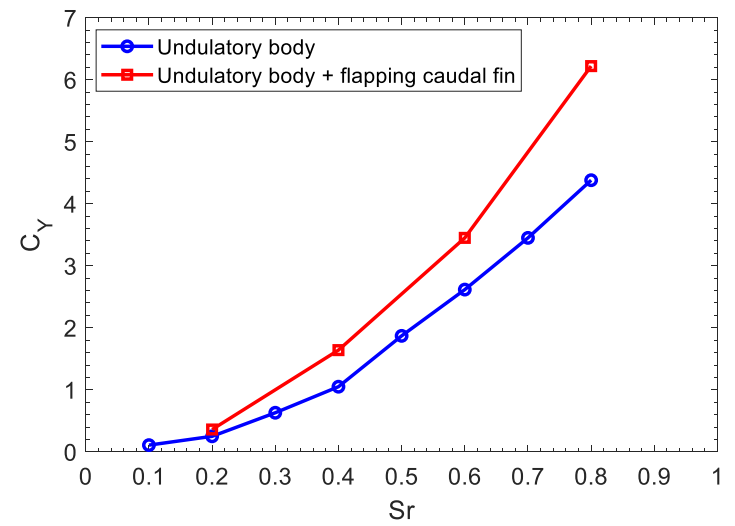

(b) Lateral side force coefficient $C_{Y}$

Fig. 17. Effect of the flapping caudal fin on (a) the drag force coefficient and (b) the lateral force coefficient. Results are obtained for varying Strouhal number values.

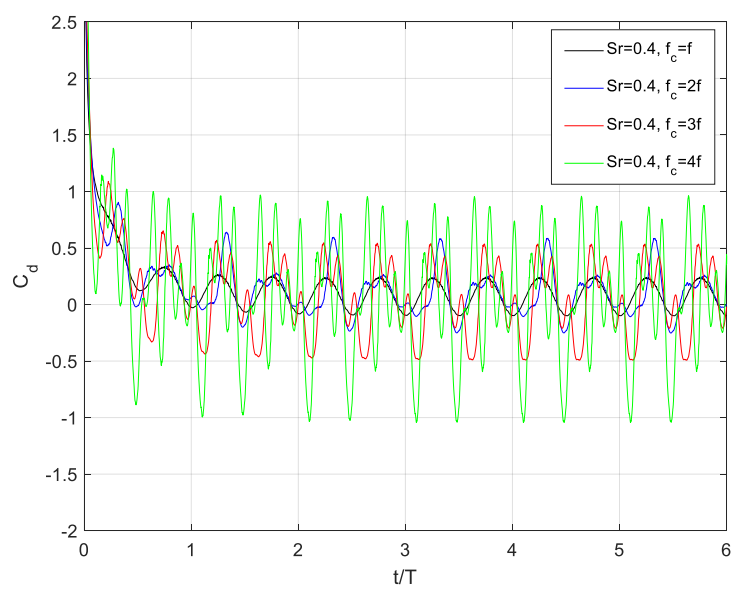

(a) Drag coefficient $C_{d}$

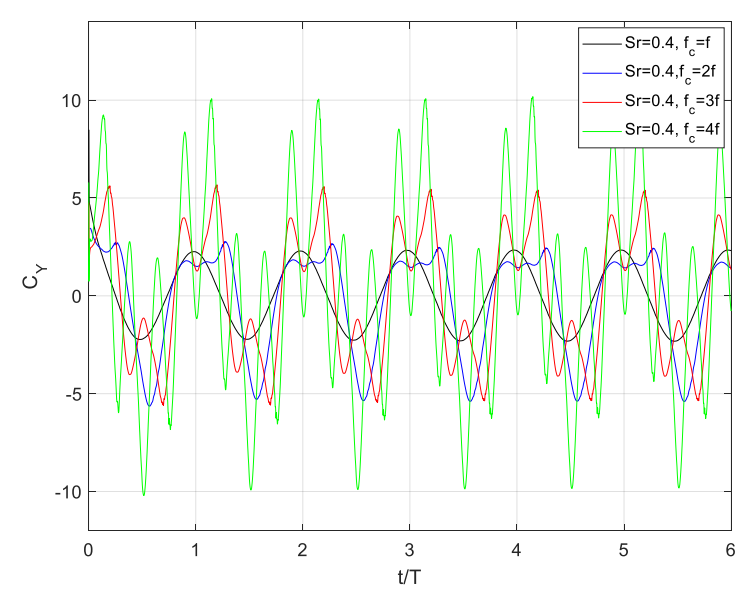

(b) Lateral side force coefficient $C_{Y}$

Fig. 18. Time histories of (a) the drag coefficient and (b) the lateral side force coefficient for varying flapping frequency of the caudal fin. The Strouhal number is set equal to 0.4 .

Next, we vary the flapping frequency of the caudal fin while maintaining the Strouhal number constant and equal to 0.4 . We note that the Strouhal number is defined in terms of the frequency of the undulatory body, which is used to demonstrate the impact of varying the frequency of the caudal fin flapping motion on the swimming performance. The frequency of the flapping motion can be considered as design component of a robotic fish. Controlling this frequency can be achieved by using servo motors. The obtained time histories of the drag and lateral side force coefficients are plotted in Fig. 18 Higher variations in the hydrodynamic loads are attained when increasing the flapping frequency. The RMS values of the drag and lateral side force coefficients increase by a factor of 5.87 and 4.39, respectively, when increasing the flapping frequency of the caudal fin $f_{c}$ from $f$ to $4 f$. A slight change in the mean values of the unsteady hydrodynamic loads is found for varying $f_{c}$ while keeping $S_{r}$ constant. This is true except the case $f_{c}=4 f$ in which we observed a noticeable improvement in the thrust generation. 


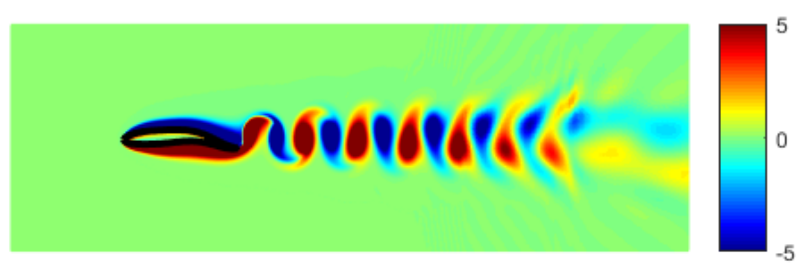

(a) Without caudal fin

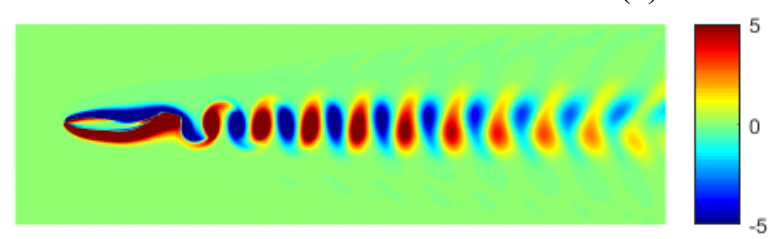

(b) $f_{c}=f$

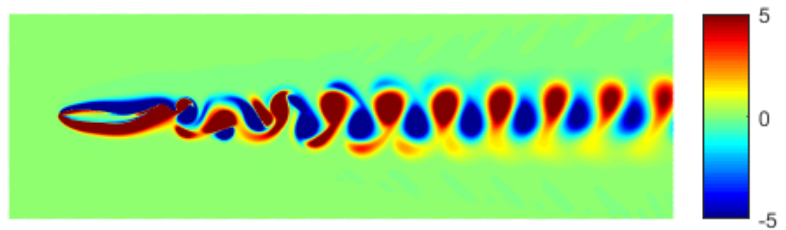

(d) $f_{c}=3 f$

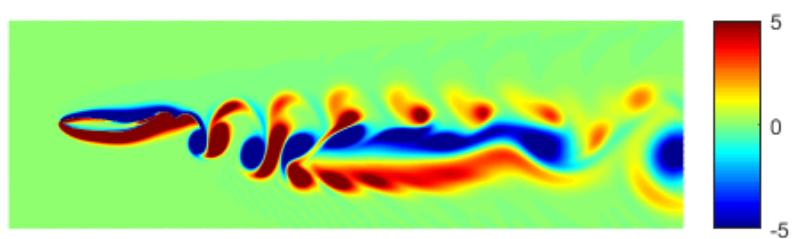

(c) $f_{c}=2 f$

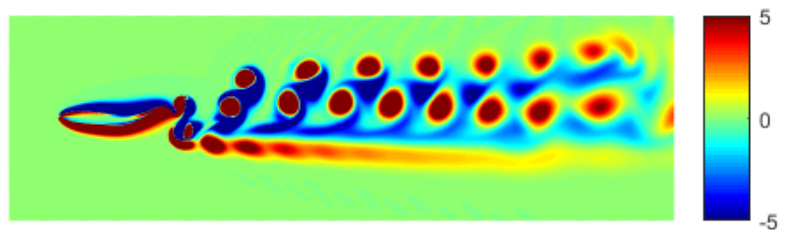

(e) $f_{c}=4 f$

Fig. 19. Vorticity pattern of the wakes generated by the fish-like locomotion with and without flapping caudal fin at $R_{e}=500$ and $S_{r}=0.4$ for different flapping frequency. The frequency of the undulatory body is kept constant while varying only the flapping frequency of the caudal fin.

Fig 19. displays the vorticity pattern of the wakes generated by the fish-like locomotion with flapping caudal fin at $R_{e}=500$ when varying the flapping frequency. The Strouhal number is kept constant and equal to 0.4 . The results demonstrate the impact of the caudal fin movement on the wake structure. The combination of the undulatory locomotion of the fish body and the flapping of the caudal fin while setting $f_{c}=f$ results in two layers of alternating vortices propagating in one row. This pattern is similar to that obtained for the case with only undulatory body (see Figs. 19(a) and 19(b)). As the $f_{c}$ increases (i.e., speed up the flapping motion of the caudal fin), the two layers of alternating vortices move away from each other. Interestingly, setting $f_{c}=4 f$ leads to the transition from the aligned arrangement of vortices of opposite signs to a deflected wake. The symmetry of the wake structure is broken and we observe the formation of pairs of positive vortices with a single vortex of negative vorticity in between. This vorticity pattern produces a momentum surfeit in the near wake region (jet flow) resulting in thrust production unlike the case of $f_{c}=f$ in which the combined undulatory body and flapping caudal fin experienced positive drag force when $S_{r}=$ 0.4 as shown in Fig. 18(a). These simulation results demonstrate that further enhancement in the swimming performance can be achieved by controlling the frequency of the flapping motion of the caudal fin.

\subsection{Effects of rigid pectoral fins}

A more realistic representation of the fish morphology includes rigid pectoral fins that are attached to the undulatory body. As such, we investigate the impact of such fins on the hydrodynamic performance of the fish. We consider two cross sections of the actual three-dimensional fish body at different positions along the spanwise direction as shown in Fig. 20(a). We consider two- 
dimensional representations as displayed in Fig. 20(b). The central part (Section A) intersects the fish body, the caudal fin and the pectoral fins while the upper part (Section B) does not include the pectoral fins. Section B corresponds to the simulation cases presented above. The pectoral fins are given a similar NACA profile to that of the caudal fins with a chord length of $15 \%$ of the total body length. They are placed at a distance $d_{p}$ from the leading edge (fish nose) and assumed to move like rigid surfaces while following the undulatory motion of the main body.

Side view

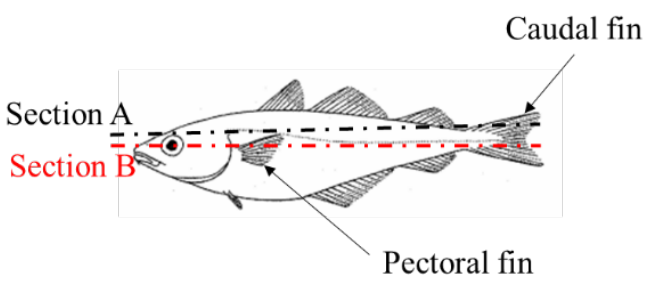

(a)
Top view

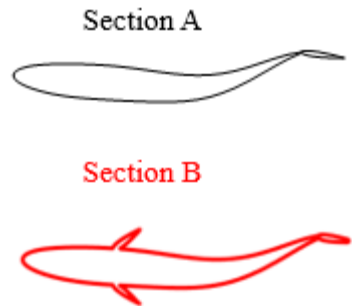

(b)

Fig. 20. Illustrative schematic of fish model: (a) Three-dimensional actual fish, (b) Twodimensional representations of the undulating fish with rigid pectoral fins as simulated.

In the subsequent simulations, the computational domain is discretized using 858,496 nodes, with local refinement around the immersed fish-like body. The spacing of the finest grid around the immersed object is $h_{\text {ref }}=0.0028125$. The time step is estimated based on Gershgorin theorem as described in [23] and is set equal to $d t=5 \times 10^{-4}$. Similar to the previous simulations, the total simulation time is set to six times the period of undulatory motion, resulting in 12,000 time steps.

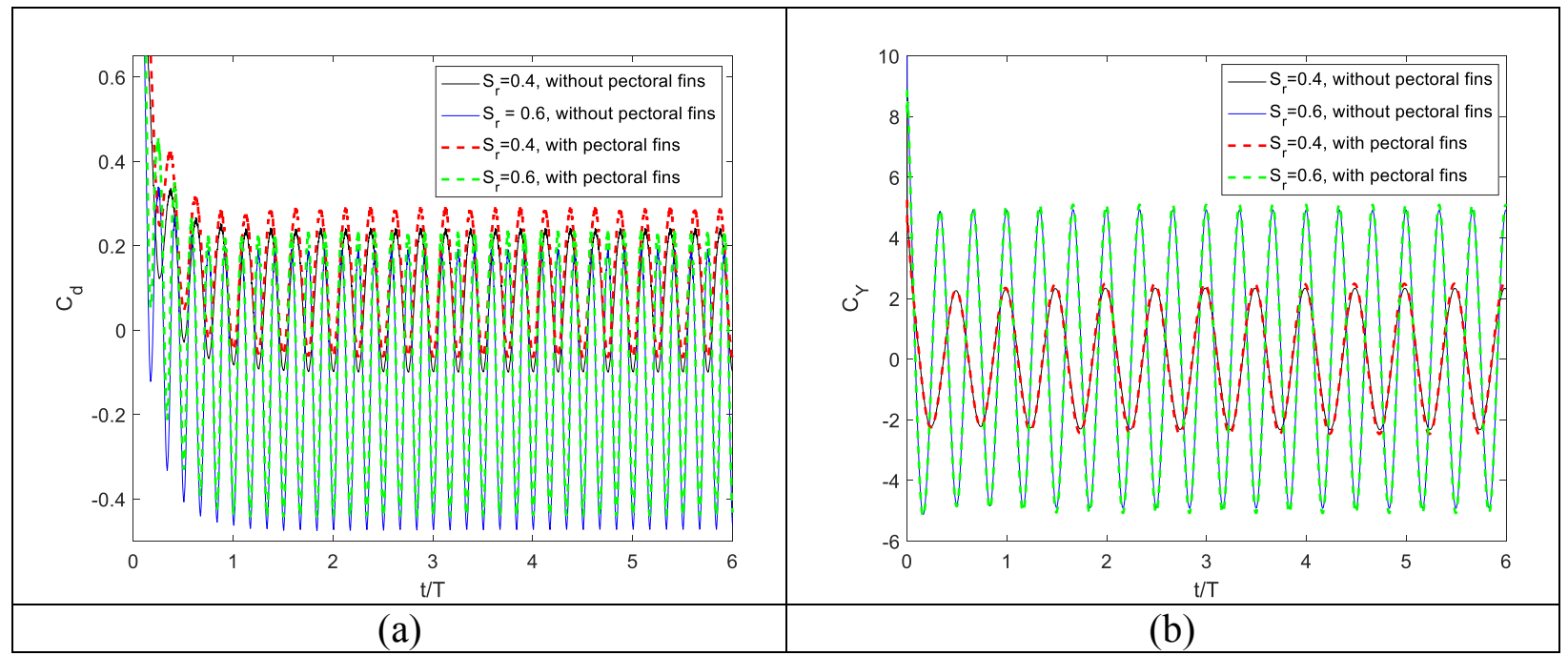

Fig. 21. Time histories of (a) the drag coefficient and (b) the lateral side force coefficient on undulating fish with and without pectoral fins for varying Strouhal number.

We show in Figs. 21(a) and 21(b) the transient variations of the drag and lateral side force coefficients, respectively, for different Strouhal numbers. The results are obtained with and without pectoral fins while keeping the flapping frequency of the caudal fin $f_{c}$ equal to the frequency of the lateral oscillations $f$. The amplitude of the flapping angle $\theta_{\max }$ is set equal to $15^{\circ}$ and the phase angle $\varphi$ is taken equal to zero. The 
pectoral fins are placed along the main fish body at a distance $d_{p}=0.3 \mathrm{l}$ from the leading edge. The mean value of the drag coefficient is found to incease from 0.07 to 0.1146 and from -0.1445 to -0.106 for $S_{r}=$ 0.4 and $S_{r}=0.6$, respectively, when adding the rigid pectoral fins. A slight increase of 3-6\% in the RMS values of the lateral side force coefficient is also observed when placing the pectoral fins at the same location.

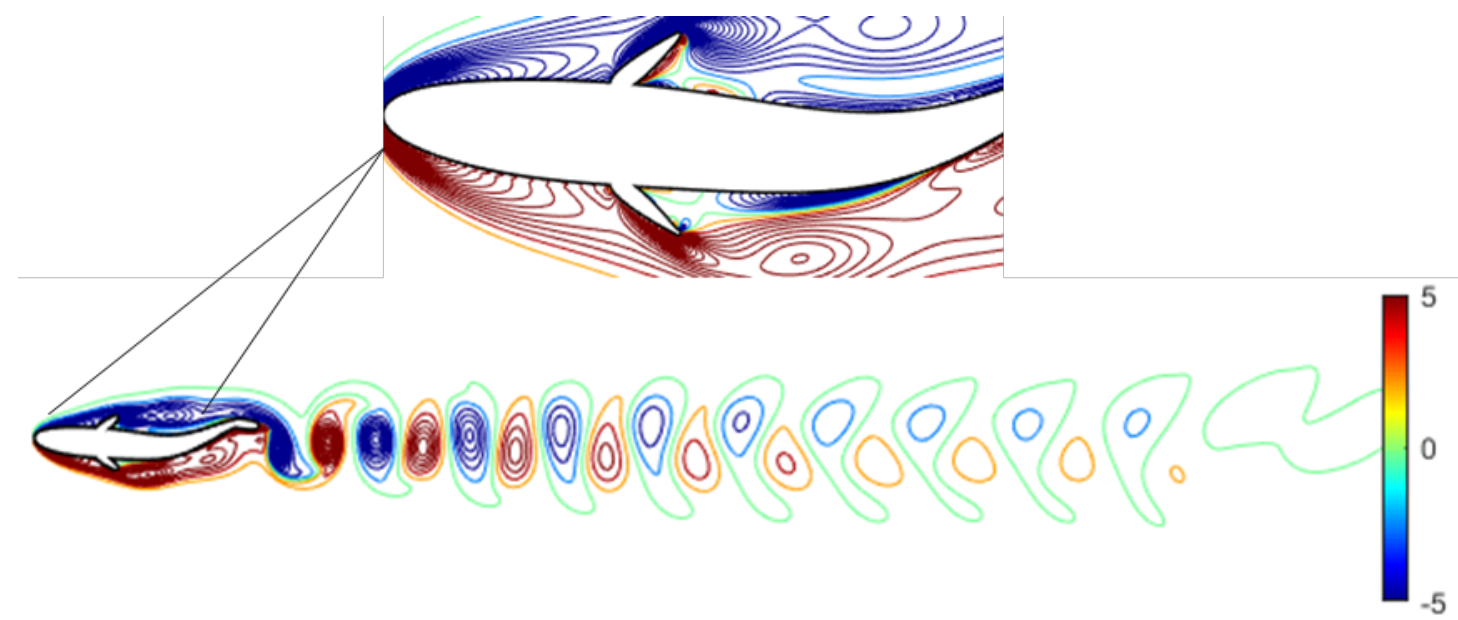

Fig. 22. Vorticity contours generated by the fish-like locomotion with flapping caudal fin and rigid pectoral fins at $R_{e}=500$ and $S_{r}=0.4$. The upper figure shows details of the vorticity distribution around the pectoral fins.

We display in Fig. 22 the vorticity contours generated by the fish-like locomotion with flapping caudal fin and rigid pectoral fins at $R_{e}=500$ and $S_{r}=0.4$. We also show details of the vorticity distribution around the pectoral fins. . The addition of rigid pectoral fins (following the undulatory motion of the main body) results in significant flow separation; that is, the detachment of the fluid flow from the fish body surface when reaching the pectroial fins and taking the forms of vortices. This results in the observed increase in the drag value.

\section{Conclusions}

We combined the immersed boundary method with meshless technique to solve the vorticity streamfunction formulation of the incompressible Navier-Stokes equations and compute the fluid forces around moving/deforming bodies. The objective is to simulate fish-like swimming and investigate the hydrodynamic performance of undulatory body with and without flapping caudal fin over a range of Strouhal numbers. The numerical method used was validated by comparing the generated unsteady drag and lateral side force coefficients and the wake structures to those reported in the literature. The simulation results showed the major impact of the Strouhal number on the vorticity pattern of the wake and thrust generation. The analysis of the effect of the lateral oscillations of the tail on the hydrodynamic performance revealed the existence of an optimal lateral oscillations amplitude that minimizes the Strouhal number needed to reach thrust-based propulsion. A significant improvement in the thrust generation is obtained when incorporating a flapping caudal fin. The production of positive thrust is observed to occur at a lower value of the Strouhal number when including the caudal fin. Furthermore, controlling the flapping frequency of the caudal fin was found to enable further enhancement in the hydrodynmic performance. This enhancement is associated with the transition from the aligned arrangement of alternating vortices to a 
deflected wake producing a momentum surfeit in the near wake region. The present numerical analysis demonstrates the benefit of the caudal fin movement to achieve superior swimming performance.

We also investigated the impact of rigid pectoral fins on the generation of hydrodynamic loads resulting from the undulatory motion of the fish body. The addition of rigid pectoral fins was observed to reduce the thrust production. We note that the current study was concerned with two-dimensional hydrodynamic simulations because of the impracticality of performing large number of three-dimensional test cases to conduct all parametric and sensitivity analysis presented here. Yet, the conclusions drawn can inform threedimenisional simulations and experimental tests of robotic fish. Another limitation of the present numerical investigation is the flexibility of the pectoral fins which has not been taken into consideration. In fact, a live fish flaps its pectoral fins to maneuvre its swimming.

\section{Acknowledgment}

This work was supported by the American University of Sharjah Faculty research grant FRG19-M-E26 (fund number EN6001). G. C. Bourantas, K. Miller, and A. Wittek acknowledge funding of the Australian Government through the Australian Research Council ARC Discovery Project Grant DP160100714.

All numerical algorithms used in this study were developed at the Intelligent Systems for Medicine Laboratory of the University of Western Australia.

\section{References}

[1] Cai Y. R., Bi S. S., Zheng L. C., 2010, Design and experiments of a robotic fish imitating cownose ray. Journal of Bionic Engineering, 7(2), 120-126.

[2] Valdivia y Alvarado P., 2007, Design of biomimetic compliant devices for locomotion in liquid environments, $\mathrm{PhD}$ Thesis. MIT, Cambridge

[3] Valdivia y Alvarado P., Youcef-Toumi K., 2006, Design of machines with compliant bodies for biomimetic locomotion in liquid environments. ASME Journal of Dynamic Systems, Measurements, and Control, 128, 3-13.

[4] Chen, Z., Shatara, S., Tan, X., 2010. Modeling of biomimetic robotic fish propelled by an ionic polymermetal composite caudal fin. IEEE/ASME Transaction Mechatronics, 15(3), 448-459.

[5] Yu J., Chen S., Wu Z., and Wang W., 2016, On a miniature free-swimming robotic fish with multiple sensors. International Journal of Advanced Robotic Systems, 13:62. doi: 10.5772/62887

[6] Xie G., Wang L., and Hu Y., 2015, Multiple Autonomous Robotic Fish Collaboration. Robot Fish: Bioinspired Fishlike Underwater Robots. Springer.

[7] Zhang, S., Qian, Y., Liao, P., Qin, F., Yang, J., 2016. Design and control of an agile robotic fish with integrative biomimetic mechanism. IEEE/ASME Transactions Mechatronics, 21(4), 1846-1857.

[8] Beardsley, C., Bergeron, L., McLean, A., Nguyen, K., Vu, M., Watson, C., Nayfeh, A., Shehata, H., Woolsey, C. and Hajj, M., 2018, October. A Modular Biolocomotion Emulator for Hydrodynamic Testing in a Towing Tank. In OCEANS 2018 MTS/IEEE Charleston (pp. 1-8). IEEE. 
[9] Du, R., Li, Z., Youcef-Toumi, K., and Alvarado, P. V., Robot Fish Bio-inspired Fishlike Underwater Robots. Springer. 2015

[10] Salazar, R., Fuentes, V., and Abdelkefi, A., 2018, Classification of biological and bioinspired aquatic systems: A review. Ocean Engineering, 148, 75-114.

[11] Zhong Y., Song, J., Yu, H., and Du, R., (2018) A Study on Kinematic Pattern of Fish Undulatory Locomotion Using a Robot Fish, Journal of Mechanisms and Robotics 10:041013-1.

[12] Bergmann, M. and Iollo, A., 2011, Modeling and simulation of fish-like swimming. Journal of Computational Physics, 230, 329-348.

[13] Li, N., Liu, H., Su, Y., 2017, Numerical study on the hydrodynamics of thunniform bio-inspired swimming under self-propulsion. PLoS ONE $12(3)$ : e0174740. https://doi.org/10.1371/journal.pone.0174740

[14] Khalid, M. S. U., Akhtar, I., and Dong, H., 2016, Hydrodynamics of a tandem fish school with asynchronous undulation of individuals. Journal of Fluids and Structures, 66, 19-35.

[15] Wen, L., Liang, J., Shen, Q., Bao, L., and Zhang, Q., 2013, Hydrodynamic performance of an undulatory robot: functional roles of the body and caudal fin locomotion. International Journal of Advanced Robotic Systems, 10, 1-10.

[16] Khalid, M. S. U., Akhtar, I., Imtiaz, H., Dong, H., and Wu, B., 2018, On the hydrodynamics and nonlinear interaction between fish in tandem configuration. Ocean Engineering, 157, 108-120.

[17] Ghaffari, S. M., Viazzo, S., Schneider, K., and Bontoux P., 2015, Simulation of forced deformable bodies interacting with two-dimensional incompressible flows: Application to fish-like swimming. International Journal of Heat and Fluid Flow, 51, 88-109.

[18] Xia, D., Chen, W-S., Liu, J-K., and Wu, Z., 2016, Effect of head swing motion on hydrodynamic performance of fishlike robot propulsion. Journal of Hydrodynamics, Ser. B, 28(4), 637-647.

[19] Zhao, Z. and Dou, L., 2019, Effects of the structural relationships between the fish body and caudal fin on the propulsive performance of fish. Ocean Engineering, 86, 106-117.

[20] Gao, A. and Triantafyllou, M. S., 2018, Independent caudal fin actuation enables high energy extraction and control in two dimensional fish-like group swimming. Journal of Fluid Mechanics, 850, 304-335.

[21] Maertens, A. P., Gao, A., and Triantafyllou, M. S., 2017, Optimal undulatory swimming for a single fish-like body and for a pair of interacting swimmers. Journal of Fluid Mechanics, 813, 301-345.

[22] Bergmann, M., Iollo, A., and Mittal, R., 2014, Effect of caudal fin flexibility on the propulsive efficiency of a fish-like swimmer. Bioinspiration \& Biomimetics, 9(4), 046001

[23] Bourantas, G. C., Zwick, B. F., Joldes, G. R., Loukopoulos, V. C., Tavner, A. C. R., Wittek, A., and Miller, K., 2019, An explicit meshless point collocation solver for incompressible Navier-Stokes equations. $\operatorname{arXiv}$ https://arxiv.org/abs/1906.05387, also available on ISML Reports as ISML Research Report \# 01/2019 (http://isml.ecm.uwa.edu.au/ISML/index.php/Archived_Reports). 
[24] Bourantas, G. C., Loukopoulos, V. C., Joldes, G. R., Wittek, A., and Miller, K., 2019, An explicit meshless point collocation method for electrically driven magnetohydrodynamics (MHD) flow. Applied Mathematics and Computation, 348, 215-233.

[25] Ren, W. W., Wu, J., Shu, C., and Yang, W. M., 2012, A stream function-vorticity formulation-based immersed boundary method and its applications. International Journal for Numerical Methods in Fluids, $70,627-645$.

[26] Schrader, B., Reboux, S., and Sbalzarini. I. F., 2010, Discretization correction of general integral PSE operators for particle methods. Journal of Computational Physics, 229, 4159-4182.

[27] Bourantas, G. C., Cheesman, B. L., Ramaswamy, R., and Sbalzarini. I. F., 2016, Using DC PSE operator discretization in Eulerian meshless collocation methods improves their robustness in complex geometries. Computers and Fluids, 136, 285-300.

[28] Lai, M. C., and Peskin. C. S., 2000, An immersed boundary method with formal second-order accuracy and reduced numerical viscosity. Journal of Computational Physics, 160, 705-719.

[29] Degond, P. and Mas-Gallic, S., 1989, The weighted particle method for convection-diffusion equations. Part 2: The anisotropic case. Mathematics of Computation, 53(188), 509-525.

[30] Eldredge, J. D., Leonard, A., and Colonius, T., 2002, A general deterministic treatment of derivatives in particle methods. Journal of Computational Physics, 180(2), 686-709.

[31] Schrader. B., Discretization-corrected PSE operators for adaptive multiresolution particle methods. Ph.D. thesis, Diss., Eidgenossische Technische Hochschule ETH Zurich, No. 19566, 2011.

[32] Dütsch, H., Durst, F., Becker, S., and Lienhart. H., 1998, Low-Reynolds-number flow around an oscillating circular cylinder at low Keulegan-Carpenter numbers. Journal of Fluid Mechanics, 360, 249271.

[34] Yang, J. and Balaras, E., 2006 An embedded-boundary formulation for large-eddy simulation of turbulent flows interacting with moving boundaries. Journal of Computational Physics, 215, 12-40.

[35] Wang, Z. L., Fan, J. R., and Cen, K. F., 2009, Immersed boundary method for the simulation of 2D viscous flow based on vorticity-velocity formulations. Journal of Computational Physics, 228, 1504-1520.

[36] Lee, J., Kim, J., Choi, H., and Yang, K., 2011, Sources of spurious force oscillations from an immersed boundary method for moving-body problems. Journal of Computational Physics, 230, 2677-2695.

[37] Khalili, M. E., Larsson, M., and Müller, B., 2018, Immersed boundary method for viscous compressible flows around moving bodies. Computers and Fluids, 170, 77-92.

[38] Xu, S. and Wang, Z.J., 2006, An immersed interface method for simulating the interaction of a fluid with moving boundaries. Journal of Computational Physics, 216, 454-493.

[39] M. Gazzola, A. A.Tchieu, D. Alexeev, A.de Brauer, P.Koumoutsakos., 2016, Learning to school in the presence of hydrodynamic interactions. Journal of Fluid Mechanics, 789,726-749.

[40] H. S. Jiang, T. R. Osborn, C. Meneveau., 2002, The flow field around a freely swimming copepod in steady motion. Part I. Theoretical analysis. J. Plankton Res. 24, 167-189. 